\title{
Efficient two-dimensional compressive sensing in MIMO radar
}

\author{
Nafiseh Shahbazi ${ }^{1 *}$, Aliazam Abbasfar ${ }^{1}$ and Mohammad Jabbarian-Jahromi ${ }^{2}$
}

\begin{abstract}
Compressive sensing (CS) has been a way to lower sampling rate leading to data reduction for processing in multiple-input multiple-output (MIMO) radar systems. In this paper, we further reduce the computational complexity of a pulse-Doppler collocated MIMO radar by introducing a two-dimensional (2D) compressive sensing. To do so, we first introduce a new 2D formulation for the compressed received signals and then we propose a new measurement matrix design for our $2 \mathrm{D}$ compressive sensing model that is based on minimizing the coherence of sensing matrix using gradient descent algorithm. The simulation results show that our proposed 2D measurement matrix design using gradient decent algorithm (2D-MMDGD) has much lower computational complexity compared to one-dimensional (1D) methods while having better performance in comparison with conventional methods such as Gaussian random measurement matrix.
\end{abstract}

Keywords: Compressive sensing, Measurement matrix, Multiple-input multiple-output (MIMO) radar, Sensing matrix, Two-dimensional sparse signal model

\section{Introduction}

Compressive sensing (CS) is a signal processing method for reconstructing a signal that is sparse in a specific domain $[1,2]$. In the past two decades, much research in various disciplines such as mathematics, statistics, signal processing, and communication systems has been conducted in CS topic in order to exploit its advantages for a wide range of applications. For example, analogto-information conversion [3], remote sensing [4], channel estimation in the communication systems [5], medical imaging [6], and image reconstruction [7] are some of these applications.

In CS, the main goal is to find the sparsest vector $s$ that satisfies an underdetermined system of linear equations $y=\Phi \Psi s$, in which the number of variables is much larger than the number of equations, where $y$ is the measurement vector, $\boldsymbol{\Phi}$ is the measurement matrix, and $\Psi$ is the basis matrix. It can be formulated in mathematic language as, minimize $\|\boldsymbol{s}\|_{0}$, subject to $\boldsymbol{y}=\boldsymbol{\Phi} \boldsymbol{\Psi} \boldsymbol{s}$, where $\|\cdot\|_{0}$ is $l_{0}$-norm and $\boldsymbol{A}=\boldsymbol{\Phi} \boldsymbol{\Psi}$ is called the sensing matrix. The $l_{0}$-norm calculates the number of non-zero

\footnotetext{
* Correspondence: n.shahbazi@ut.ac.ir

'School of Electrical and Computer Engineering, University of Tehran, Tehran, Iran

Full list of author information is available at the end of the article
}

components of a vector. To solve this problem, we need a combinatorial search to find the minimum $l_{0}$-norm, which is an NP-hard problem. One of the solutions proposed for this problem is to replace $l_{0}$-norm with $l_{1}$-norm and convert the problem to a convex one. A famous algorithm that minimizes $l_{1}$-norm is basis pursuit (BP) [8].

Some applications of compressive sensing in radar systems have been recently studied in [9-11]. In [12], the direction of arrival (DOA) of the signal is estimated using CS for communication systems. In order to estimate the desired parameters in CS radar, it should be assumed that the number of targets to be found is much smaller than the whole number of radar bins, which is the case in most practical radar applications.

Multiple-input multiple-output (MIMO) radar systems have received the attention of many researchers in recent years. There are two different types of MIMO radar systems which are categorized according to their antennas configuration. In the first type, the antennas are widely separated from each other relative to their distance to the target [13]. In the second one, which is considered in this paper, the antennas are collocated and located close to each other [14]. 
In [15], the sparse signal model of MIMO radar is derived using only one incoming pulse. Furthermore, a sparse learning via iterative minimization (SLIM) algorithm is developed and compared to other sparse methods such as iterative adaptive approach (IAA) [16]. The main drawback of SLIM algorithm in [15] is that its computational complexity will be very high for pulse-Doppler MIMO radars.

Recently, a new signal model with two-dimensional (2D) sparse parameters, called 2D sparse signal model, has been introduced [17, 18], and some algorithms for 2D sparse reconstruction have been proposed [19-24]. Specifically, in [19], the 2D version of IAA is derived in which its computational cost is drastically reduced in comparison with the one-dimensional (1D) IAA. The other 2D sparse recovery algorithm is the smoothed L0 (SL0) algorithm that minimizes an approximated $l_{0^{-}}$ norm function [20] and has much lower computational complexity than its 1D counterpart. In [21], a 2D sparse signal model for a radar is obtained and solved by 2DSL0 algorithm with acceptable results. Also, 2D-SLIM [22], 2D Truncated Newton Interior Point Method (2DTNIPM) [23], and 2D Sparse Bayesian Learning using Laplace Prior (2D-SBL-LP) [24] have been proposed for pulse Doppler MIMO radars. These papers have demonstrated that the $2 \mathrm{D}$ proposed algorithms have much less computational complexity compared to corresponding 1D sparse recovery algorithms.

The main goal of sparsity-based methods for MIMO radar systems $([21-24])$ is to achieve accurate estimates for target parameters, whereas in this paper, we mainly focus on the CS MIMO radar problem to reduce the sampling rate lower than the Nyquist criterion by designing a suitable measurement matrix. Therefore, the main difference between our 2D CS MIMO radar signal model and 2D sparse model in [21-24] is that we consider the measurement matrices in our model in which these measurement matrices can be applied on receivers and received pulses, separately.

In [18], a 2D CS signal model has been proposed for inverse synthetic aperture radar (ISAR) imaging radar in which a random sub-sampling in both range and azimuth dimensions is utilized. Also, a 2D CS image reconstruction algorithm based on iterative gradient projection is derived in [25].

Compressive sensing for MIMO radar systems is proposed and analyzed in $[26,27]$. A MIMO radar, which is one of the most practical applications of MIMO systems, transmits some independent waveforms by its transmit antennas and has superior spatial resolution compared to traditional radar systems. CS in MIMO radar has the ability to achieve the same localization performance as the traditional methods while using much lower number of measurements, which is achieved by applying a measurement matrix to the normally measured samples.

In CS, the measurement matrix has a key role in the performance of sparse signal recovery algorithm. Therefore, we can improve the performance of target detection in CS MIMO radar by designing a suitable measurement matrix. The conventional approach for choosing this matrix is a Gaussian random measurement matrix (GRMM) which is not necessarily the best one for CS. According to [28], if the mutual coherence (MC) that is the maximum value of pairwise correlation among the columns of $\boldsymbol{A}$ is small, the sparse signal can be recovered with high probability. Recently, some measurement matrix design methods have been proposed based on minimizing the MC of the sensing matrix [29-31]. Elad [29] designed the measurement matrix based on mutual coherence minimization using a shrinkage operation. Duarte-Carvajalino [30] optimized the measurement matrix and basis matrix jointly by a KSVDbased algorithm. However, these methods are adapted for real signals and also have many parameters that should be set properly.

In [31], a gradient descent method is used to minimize the MC of $\boldsymbol{A}$ which is described as the absolute offdiagonal elements of the Gram matrix. It is shown that this method can achieve higher sparse reconstruction performance compared to previous methods. In our paper, we extend this method to a complex CS MIMO radar signal and decrease the computational complexity by proposing a 2D CS MIMO radar signal model. In [32], a method for optimizing the measurement matrix of MIMO radar systems is proposed based on two different criterions; first one is to minimize the summation of coherence of cross columns in the sensing matrix plus maximize the signal-to-interference ratio (SCSM + SIR), and the second criterion is to maximize SIR by imposing a special structure on the measurement matrix. However, both methods are suboptimal solutions and may not have acceptable performance in different situations.

In this paper, we introduce a 2D signal model in CS for a collocated MIMO radar with point targets, and then we improve the efficiency of this 2D MIMO radar model by proposing a measurement matrix design using gradient decent algorithm (MMDGD) in which the MC of sensing matrix is minimized. We call the proposed method as 2D-MMDGD.

The gradient descent algorithm is popular for very large-scale optimization problems due to its simplicity of the implementation and its low computational load. Although our proposed 2D-MMDGD method leads to a nonlinear problem and there is no guarantee that we find its global minimum, its performance is always better than GRMM. The reason is that we use GRMM as an initial value of our proposed algorithm; therefore, the designed matrix will have less mutual coherence and 
consequently better performance compared to its initial value, i.e., GRMM. Simulation results show that our proposed 2D-MMDGD performs much better than GRMM, SIR, and SCSM + SIR methods.

The rest of the paper is organized as follows. Sections 2 and 3 describe the 1D and 2D CS signal model of the MIMO Radar, respectively. In Section 4, we propose a measurement matrix design for $1 \mathrm{D}$ and $2 \mathrm{D}$ CS model using gradient decent algorithm. The computational complexity of the proposed methods is discussed in Section 5. Simulation results are given in section 6. Finally, we have conclusions in section 7 .

Notations: Lower case and capital letters in bold denote vectors and matrices. Superscripts $(.)^{T},(.)^{H}$ denote the transpose and Hermitian transpose of a matrix, respectively. $\mathbf{0}_{M \times N}$ denotes an $M \times N$ matrix with all zero elements and $\boldsymbol{I}_{N}$ denotes an $N \times N$ identity matrix. Also, $\|$. $\|_{2}$ and $\|.\|_{F}$ denote the square and Frobenius norm of a vector/matrix, respectively. The operator $\otimes, \operatorname{var}($.$) , and$ $E($.$) are the Kronecker product, variance, and expect-$ ation of a random variable, respectively.

\section{1D-CS signal model of MIMO radar}

In this section, we describe the 1D signal model of a CS pulse-Doppler MIMO radar. In this model, we assume that one period of Doppler frequencies of targets spans a duration of several received pulses. It means that $f_{\mathrm{d}} \tau \ll 1$, where $f_{\mathrm{d}}$ and $\tau$ are the Doppler shift frequency and the pulsewidth, respectively. If $f_{\mathrm{c}}$ is the carrier frequency, this assumption can be written as $f_{\mathrm{r}} \ll B \ll f_{\mathrm{c}}$, where $f_{r}$ is the radar pulse repetition frequency (PRF) and $B$ is the bandwidth of the transmitted signal. Based on this assumption, the Doppler effect on each pulse is simply a phase shift. We assume that Doppler shift frequency is in the interval $\left[\frac{-f_{\mathrm{r}}}{2}, \frac{f_{\mathrm{r}}}{2}\right)$ and the targets are located behind the maximum unambiguous range. Suppose that the Doppler frequency of interest divided into $N_{\mathrm{d}}$ bins:

$$
f_{\mathrm{d}}=-\frac{f_{\mathrm{r}}}{2}+\frac{f_{\mathrm{r}}(d-1)}{N_{\mathrm{d}}}
$$

where $d$ is the Doppler index and $d=1,2, \cdots, N_{\mathrm{d}}$. The Doppler phase shift over one pulse period for the $d$ th Doppler bin is obtained as

$$
\theta_{\mathrm{d}}=\frac{2 \pi f_{\mathrm{d}}}{f_{\mathrm{r}}}
$$

The transmitted signal samples of all antennas can be put together in a matrix as

$$
\boldsymbol{V}=\left[\boldsymbol{v}_{1} \cdots \boldsymbol{v}_{M_{t}}\right]
$$

where $\boldsymbol{v}_{i} \in \mathbb{C}^{L \times 1}, \quad i=1, \ldots, M_{\mathrm{t}}$ is the samples of the transmitted signal with length $L$ by the $i$ th antenna (for a total of $M_{\mathrm{t}}$ transmit antennas).

Without loss of generality, a uniform linear array for the transmit and receive antenna arrays has been used in our model and simulations in this paper.

Suppose that the number of range bins is $N_{\mathrm{r}}$ in the radar surveillance area, then the largest possible delay between the transmit and receive pulses is $\left(N_{\mathrm{r}}-1\right)$.

We consider the number of the angle bins of the antenna array to be $N_{\mathrm{a}}$ and the angle bins are $\left\{\boldsymbol{\alpha}_{\boldsymbol{a}}\right\}_{a=1}^{N_{\mathrm{a}}}$. The transmit and receive antenna array steering vectors of the $\boldsymbol{a}$ th angle bin are shown, respectively, as $\boldsymbol{a}_{\boldsymbol{a}} \in C^{M_{t} \times 1}$, $\boldsymbol{b}_{\boldsymbol{a}} \in C^{M_{r} \times 1}$.

$$
\boldsymbol{a}_{a}=\left[\begin{array}{llll} 
& e^{-\frac{j 2 \pi \Delta_{t} \sin \left(\alpha_{a}\right)}{\lambda_{0}}} & \cdots & e^{-\frac{j 2 \pi\left(M_{t}-1\right) \Delta_{t} \sin \left(\alpha_{a}\right)}{\lambda_{0}}}
\end{array}\right]^{T}
$$

and

$$
\boldsymbol{b}_{a}=\left[\begin{array}{lll} 
& e^{-\frac{j 2 \pi \Delta_{r} \sin \left(\alpha_{a}\right)}{\lambda_{0}}} \cdots & e^{-\frac{j 2 \pi\left(M_{r}-1\right) \Delta_{r} \sin \left(\alpha_{a}\right)}{\lambda_{0}}}
\end{array}\right]^{T}
$$

where $\Delta_{\mathrm{t}}$ and $\Delta_{\mathrm{r}}$ are the distance between elements of transmit and receive antennas, respectively, and $\lambda_{0}$ is the signal of transmitting wavelength.

The compressed received signal in the $m$ th antenna returned from the $p$ th transmitted pulse can be arranged in an observation vector, $\boldsymbol{y}_{p m} \in \mathbb{C}^{M \times 1}$ as follows:

$$
\boldsymbol{y}_{p m}=\boldsymbol{\phi}_{p m} \sum_{r=1}^{N_{r}} \sum_{a=1}^{N_{a}} \sum_{d=1}^{N_{d}} s_{r, a, d} e^{j(p-1) \theta_{d}} \boldsymbol{J}_{r} \boldsymbol{V} \boldsymbol{a}_{a} e^{-\frac{j 2 \pi(m-1) \Delta_{r} \sin \left(a_{a}\right)}{\lambda_{0}}}+\boldsymbol{n}_{p m}
$$

where $\boldsymbol{\phi}_{p m} \in \mathbb{C}^{M \times \tilde{L}}$ is the measurement matrix applied on the $p$ th pulse and $m$ th receive antenna for $p=1, \ldots, N_{p}$ and $m=1, \ldots, M_{n}$ and $\widetilde{L}=L+N_{r}-1$. The parameters $M, N_{\mathrm{p}}$, and $M_{\mathrm{r}}$ are the length of observation vector (compressed observations), the number of pulses, and receive antennas, respectively. It is noted that for compressive sensing scenario, we have $M<\tilde{L}$.

The vector $\boldsymbol{n}_{p m}=\boldsymbol{\phi}_{p m} \boldsymbol{e}_{p m}$ is the additive noise for the $m$ th receive antenna and $p$ th pulse and $\boldsymbol{e}_{p m} \in \mathbb{C}^{\tilde{L} \times 1}$ is the receiver noise with complex Gaussian random distribution, with zero mean and covariance matrix $\boldsymbol{I}_{\tilde{L}}$. As it is noted, the noise covariance matrix $\boldsymbol{n}_{p m}$ is $\boldsymbol{\phi}_{p m} \boldsymbol{\phi}_{p m}^{H}$.

The matrix $\boldsymbol{J}_{r} \in \mathbb{C}^{\tilde{L} \times L}$ is a time-shift matrix which returns the shifted version of the transmitted signal for the $r$ th range bin. In fact, $\boldsymbol{J}_{r}$ time-shifts the transmitted signals and extends them up to the maximum received signal duration (by zero-padding) and it is defined as 


$$
\boldsymbol{J}_{r}=\left[\begin{array}{c}
0_{(r-1) \times L} \\
\boldsymbol{I}_{L} \\
0_{\left(N_{r}-r\right) \times L}
\end{array}\right] .
$$

Parameters $\left\{s_{r, a, d}\right\}\left(r=1, \ldots, N_{r}, a=1, \ldots, N_{a}\right.$ and $d=1$, ..., $N_{d}$ ) denote the return coefficients from targets in the radar interest area, where $s_{r, a, d}=\beta_{k}$ if the $k$ th target is located at the $(r, a, d)$ th bin and $s_{r, a, d}=0$ otherwise. In general, $\beta_{k}$ is a complex number corresponding to the radar cross section of the $k$ th target $\left(k=1,2, \ldots, N_{\mathrm{t}}\right)$, and $N_{\mathrm{t}}$ is the number of targets.

Now, the received signal samples from the $m$ th receive antenna returned from the $p$ th transmitted pulse can be written as

$$
\boldsymbol{y}_{p m}=\boldsymbol{\phi}_{p m} \boldsymbol{\psi}_{p m} \boldsymbol{s}+\boldsymbol{n}_{p m}
$$

where $\boldsymbol{s} \in \mathbb{C}^{N \times 1}$ is a sparse vector which has $N_{t} \ll N$ nonzero elements, $N=N_{r} N_{a} N_{d}$, and $\boldsymbol{\psi}_{p m}=\boldsymbol{\theta}_{p}^{T} \otimes \boldsymbol{H}_{m} \in \mathbb{C}^{\tilde{L} \times N}$ is the basis matrix for the $m$ th receiver during the $p$ th pulse, $\otimes$ is the Kronecker multiplication, and

$$
\begin{aligned}
& \boldsymbol{h}_{m(r, a)}=\boldsymbol{J}_{r} \boldsymbol{V} \boldsymbol{a}_{a} e^{\frac{j 2 \pi(m-1) \Delta r \sin \left(\alpha_{a}\right)}{\lambda_{0}} \in \mathbb{C}^{\tilde{L} \times 1},} \\
& \boldsymbol{H}_{m}=\left[\begin{array}{lll}
\boldsymbol{h}_{m(1,1)} & \boldsymbol{h}_{m(1,2)} \cdots \boldsymbol{h}_{m\left(N_{r}, N_{a}\right)}
\end{array}\right] \in \mathbb{C}^{\tilde{L} \times N_{r} N_{a}}, \\
& \boldsymbol{s}_{d}=\left[\begin{array}{llll}
s_{1,1, d} s_{1,2, d} & \cdots & s_{N_{r}, N_{a}, d}
\end{array}\right], \\
& \boldsymbol{s}=\left[\begin{array}{llll}
\boldsymbol{s}_{1} & \boldsymbol{s}_{2} & \cdots & \boldsymbol{s}_{N_{d}}
\end{array}\right]^{T}, \\
& \boldsymbol{\theta}_{p}=\left[\begin{array}{llll}
e^{j(p-1) \theta_{1}} & e^{j(p-1) \theta_{2}} & \cdots & e^{j(p-1) \theta_{N_{d}}}
\end{array}\right]^{T} \in \mathbb{C}^{N_{d} \times 1} .
\end{aligned}
$$

We can also arrange the observations for all receive antennas for the $p$ th pulse in vector form $\boldsymbol{y}_{p} \in \mathbb{C}^{M M_{r} \times 1}$ as

$$
\boldsymbol{y}_{p}=\left[\boldsymbol{y}_{p 1}{ }^{T} \cdots \boldsymbol{y}_{p M_{r}}^{T}\right]^{T}=\boldsymbol{T}_{p} \boldsymbol{\Psi}_{p} \boldsymbol{s}+\boldsymbol{n}_{p}
$$

where $\boldsymbol{n}_{p}=\boldsymbol{T}_{p} \boldsymbol{e}_{p}$ is the additive noise of all receivers and $\boldsymbol{e}_{p}=\left[\begin{array}{llll}\boldsymbol{e}_{p 1} & \cdots & \boldsymbol{e}_{p M_{r}}^{T}\end{array}\right]^{T} \quad \mathbb{C}^{\tilde{L} M_{r} \times 1}$. The matrix $\boldsymbol{T}_{p}$ is a block diagonal matrix in which the $m$ th block is a measurement matrix used for $m$ th receive antenna and defined as

$$
\boldsymbol{T}_{p}=\operatorname{blkdiag}\left(\boldsymbol{\Phi}_{p 1}, \boldsymbol{\Phi}_{p 2}, \ldots, \boldsymbol{\Phi}_{p M_{r}}\right) \in \mathbb{C}^{M M_{r} \times \tilde{L} M_{r}} .
$$

The matrix $\Psi_{p}$ in (14) which is the basis matrix for all antennas, and the $p$ th pulse is defined as

$$
\boldsymbol{\Psi}_{p}=\left[\begin{array}{c}
\boldsymbol{\psi}_{p 1} \\
\boldsymbol{\psi}_{p 2} \\
\vdots \\
\boldsymbol{\psi}_{p M_{r}}
\end{array}\right] \in \mathbb{C}^{\tilde{L} M_{r} \times N} .
$$

For all the pulses, we have

$$
\boldsymbol{y}=\left[\begin{array}{lll}
\boldsymbol{y}_{1}^{T} & \cdots & \boldsymbol{y}_{N_{P}}^{T}
\end{array}\right]^{T}=\boldsymbol{\Phi} \boldsymbol{\Psi} \boldsymbol{s}+\boldsymbol{n}
$$

where $\boldsymbol{\Phi}=\operatorname{blkdiag}\left(\boldsymbol{T}_{1}, \boldsymbol{T}_{2}, \ldots, \boldsymbol{T}_{N_{p}}\right) \in \mathbb{C}^{M M_{r} N_{p} \times \tilde{L} M_{r} N_{p}}$ is the measurement matrix which is applied to all observations in order to decrease the number of received signal samples, $\quad \boldsymbol{\Psi}=\boldsymbol{\Theta}^{T} \otimes \boldsymbol{H} \in \mathbb{C}^{\llbracket M_{r} N_{p} \times N}$ is the basis matrix for our pulse-Doppler MIMO radar system, $\boldsymbol{\Theta}=\left[\begin{array}{lll}\boldsymbol{\theta}_{1} & \cdots & \boldsymbol{\theta}_{N_{p}}\end{array}\right] \in \mathbb{C}^{N_{d} \times N_{p}}$ is the MIMO radar dictionary matrix containing the Doppler information of targets in different situations, and $\boldsymbol{n}=\boldsymbol{\Phi} \boldsymbol{e} \in \mathbb{C}^{M M_{r} N_{p} \times 1}$ is the additive noise of all receivers and pulses after compression, where $\boldsymbol{e}=\left[\begin{array}{lll}\boldsymbol{e}_{1}^{T} & \cdots & \boldsymbol{e}_{N_{p}}^{T}\end{array}\right]^{T}$. The matrix $\boldsymbol{H}$ is defined as

$$
\boldsymbol{H}=\left[\begin{array}{c}
\boldsymbol{H}_{1} \\
\boldsymbol{H}_{2} \\
\vdots \\
\boldsymbol{H}_{M_{r}}
\end{array}\right] .
$$

Generally, $\boldsymbol{H} \in \mathbb{C}^{\tilde{L} M_{r} \times N_{r} N_{a}}$ is the MIMO radar dictionary matrix containing the whole range and angle information of targets. Also, $\Psi$ can be given by:

$$
\boldsymbol{\Psi}=\left[\begin{array}{c}
\boldsymbol{\Psi}_{1} \\
\boldsymbol{\Psi}_{2} \\
\vdots \\
\boldsymbol{\Psi}_{N_{p}}
\end{array}\right]=\boldsymbol{\Theta}^{T} \otimes \boldsymbol{H}
$$

Our goal in this paper is to design the measurement matrix $\Phi$ such that the CS MIMO radar has better performance in target detection compared to conventional approaches.

To optimize $\boldsymbol{\Phi}$, four different cases can be considered:

Case 1 (general case): All sub-blocks $\boldsymbol{\phi}_{p m}\left(p=1, \ldots, N_{p}\right.$ and $\left.m=1, \ldots, M_{r}\right)$ located in $\Phi$ are different with each other, and thus, they are optimized, separately.

Case 2: We assume that the measurement matrix is equal for all receivers,

$$
\boldsymbol{\phi}_{p 1}=\boldsymbol{\phi}_{p 2}=\ldots=\boldsymbol{\phi}_{p M_{r}}=\boldsymbol{\phi}_{p} .
$$

Therefore, $\quad \boldsymbol{T}_{p}=\boldsymbol{I}_{M_{r}} \otimes \boldsymbol{\phi}_{p}$, where $\boldsymbol{\phi}_{p} \in \mathbb{C}^{M \times \tilde{L}}$ is the measurement matrix applied on the $p$ th received pulse, and $\boldsymbol{\Phi}=\operatorname{blkdiag}\left(\boldsymbol{T}_{1}, \boldsymbol{T}_{2}, \ldots, \boldsymbol{T}_{N_{p}}\right) \in \mathbb{C}^{M M_{r} N_{p} \times \tilde{L} M_{r} N_{p}}$.

Case 3: We assume that the measurement matrix is equal for all received pulses,

$$
\boldsymbol{\phi}_{1 m}=\boldsymbol{\phi}_{2 m}=\ldots=\boldsymbol{\phi}_{N_{p} m}=\boldsymbol{\phi}_{m}
$$

where $\boldsymbol{\phi}_{m} \in \mathbb{C}^{M \times \tilde{L}}$ is the measurement matrix applied on the $m$ th receive antenna; therefore, $\boldsymbol{\Phi}=\boldsymbol{I}_{N_{p}} \otimes \boldsymbol{T}$, where $\boldsymbol{T}=\operatorname{blkdiag}\left(\boldsymbol{\phi}_{1}, \boldsymbol{\phi}_{2}, \ldots, \boldsymbol{\phi}_{M_{r}}\right)$.

Case 4: We assume that the measurement matrix is equal for all receivers and pulses,

$$
\phi_{11}=\phi_{12}=\ldots=\phi_{N_{p} M_{r}}=\phi .
$$

Therefore, we have $\boldsymbol{\Phi}=\boldsymbol{I}_{N_{p}} \otimes \boldsymbol{T}$, where $\boldsymbol{T}=\boldsymbol{I}_{M_{r}} \otimes \boldsymbol{\phi}$, and $\phi \in \mathbb{C}^{M \times \tilde{L}}$ is the measurement matrix applied on all receivers and pulses. 


\section{2D-CS signal model of MIMO radar}

In this section, we derive the 2D-CS signal model for MIMO radar and explain its relationship to the classical 1D-CS signal model.

Among all cases discussed in the last section, only case 3 and case 4 can be converted to 2D model. To do so, we arrange the received signals for all pulses into a matrix as

$$
\boldsymbol{Y}=\left[\begin{array}{lll}
y_{1} & \cdots & \boldsymbol{y}_{N_{P}}
\end{array}\right]=\boldsymbol{T H} \boldsymbol{H} \Theta+\boldsymbol{N}
$$

where $\quad \boldsymbol{S}=\left[\begin{array}{llll}\boldsymbol{s}_{1}^{T} & \boldsymbol{s}_{2}^{T} & \ldots & \boldsymbol{s}_{N_{d}}^{T}\end{array}\right], \quad \boldsymbol{N}=\boldsymbol{T} \boldsymbol{E}$, and $\boldsymbol{E}=$ $\left[\begin{array}{lll}\boldsymbol{e}_{1} & \cdots & \boldsymbol{e}_{N_{P}}\end{array}\right]$. This equation is the 2D model of CS MIMO radar. We can present the relationship between 1D and 2D sparse signal model for MIMO radars transmitting a trail of pulses by using the following property [33]:

$$
\operatorname{vec}(\boldsymbol{T H} \boldsymbol{S} \boldsymbol{\Theta})=\left(\boldsymbol{\Theta}^{T} \otimes \boldsymbol{T H}\right) \operatorname{vec}(\mathbf{S})
$$

If we apply Eqs. (24) to (23), we re-derive the 1D CS model shown in Eq. (17), where we have

$$
\boldsymbol{y}=\operatorname{vec}(\boldsymbol{Y}), \boldsymbol{s}=\operatorname{vec}(\boldsymbol{S}), \boldsymbol{n}=\operatorname{vec}(\boldsymbol{N})
$$

and

$$
\boldsymbol{A}=\boldsymbol{\Phi} \boldsymbol{\Psi}=\boldsymbol{\Theta}^{T} \otimes \boldsymbol{T H} \in \mathbb{C}^{M M_{r} N_{p} \times N}
$$

Also, we can rewrite matrix $A$ as a block matrix whose $p m$ th block is the measurement matrix $\boldsymbol{\phi}_{p m}$ applied on $p$ th received pulse and $m$ th receive antenna multiplied by $p m$ th basis matrix $\boldsymbol{\Psi}_{p m}$ :

$$
\boldsymbol{A}=\left[\begin{array}{c}
\boldsymbol{A}_{11} \\
\boldsymbol{A}_{12} \\
\vdots \\
\boldsymbol{A}_{N_{p} M_{r}}
\end{array}\right]=\left[\begin{array}{c}
\boldsymbol{\phi}_{11} \boldsymbol{\psi}_{11} \\
\boldsymbol{\phi}_{12} \boldsymbol{\psi}_{12} \\
\vdots \\
\boldsymbol{\phi}_{N_{p} M_{r}} \boldsymbol{\psi}_{N_{p} M_{r}}
\end{array}\right]
$$

Figure 1 shows the block structure of the 2D-CS signal model implementation for MIMO radar. As noted before, two dictionary matrices $\boldsymbol{H}$ and $\Theta$ are constructed based on potential situations of range, angle and Doppler frequency of targets in MIMO radar surveillance area. Also, the sampling of signal is conducted based on measurement matrix $\boldsymbol{T}$ which is optimized in the following section using gradient descent algorithm.

\section{Measurement matrix design using gradient decent algorithm for CS model}

In this section, first we discuss the conditions that a measurement matrix should have, and then we propose a new measurement matrix for CS MIMO radar that is suitable for our 1D and 2D CS models.

The mutual coherence (MC) of sensing matrix $\boldsymbol{A}$ that shows the maximum value of the pairwise correlations of the column vectors of $\boldsymbol{A}$ is defined as follows [28]

$$
\mu(\boldsymbol{A})=\max _{i \neq j} \frac{\rho_{i, j}}{\left\|\boldsymbol{a}_{i}\right\|_{2}\left\|\boldsymbol{a}_{j}\right\|_{2}}
$$

where $\rho_{i, j}=\left|\boldsymbol{a}_{i}^{H} \boldsymbol{a}_{j}\right|$ and $\boldsymbol{a}_{i}$ is the $i$ th column of $\boldsymbol{A}$. The mutual incoherence property (MIP) is one of the best conditions for sparse signal reconstruction [28]. According to the MIP, for a small value of $\mu(A)$, the sparse signal can be reconstructed with high probability. In fact, the exact reconstruction of $s$ will be guaranteed if the following inequality holds [29]:

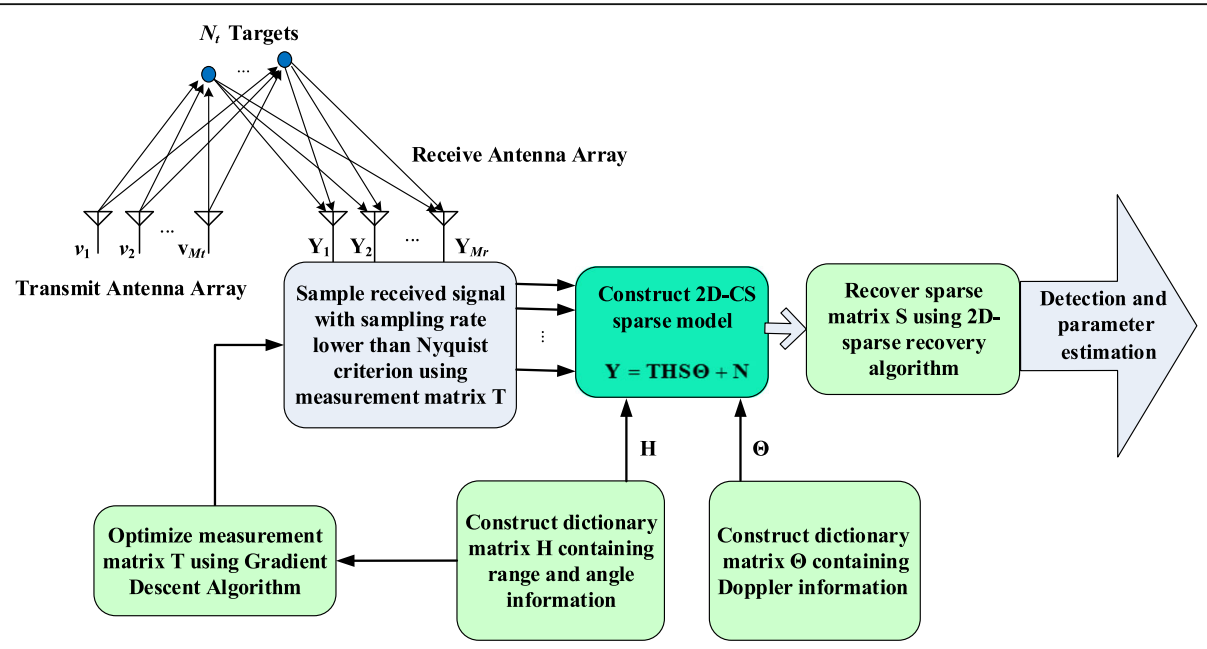

Fig. 1 Block diagram of 2D-CS signal model implementation for MIMO radar 


$$
\left\|s_{0}\right\|<\frac{1}{2}\left(1+\frac{1}{\mu(A)}\right) .
$$

The statistical analysis of MC of sensing matrix is performed in Appendix 1 for the aforementioned four cases. The following results are concluded:

$$
\begin{aligned}
& E\left(\mu_{\text {case 1 }}\right)=E\left(\mu_{\text {case } 2}\right)=E\left(\mu_{\text {case 3 }}\right)=E\left(\mu_{\text {case } 4}\right) \\
& \operatorname{var}\left(\mu_{\text {case } 1}\right) \leq \operatorname{var}\left(\mu_{\text {case } 3}\right) \leq \operatorname{var}\left(\mu_{\text {case } 4}\right) \\
& \operatorname{var}\left(\mu_{\text {case } 1}\right) \leq \operatorname{var}\left(\mu_{\text {case } 2}\right) \leq \operatorname{var}\left(\mu_{\text {case } 4}\right) .
\end{aligned}
$$

As it is seen, case 1 has the least variance of $M C$, and therefore, it is expected that it has better performance compared to other cases. On the other hand, case 4 has the biggest variance of $\mathrm{MC}$, and therefore, it is expected that it has the worst performance.

Also, as shown in [34], (28) might not have a good behavior for the case that coherence of cross columns is small. In [29], Elad proposes the average mutual coherence as an alternative criterion because of its lower computational complexity compared to (28). Therefore, the new measurement matrix optimization can be expressed as:

$$
\hat{\boldsymbol{\Phi}}=\arg \min _{\boldsymbol{\Phi}}\left\|\boldsymbol{G}-\boldsymbol{I}_{N}\right\|_{F}^{2}
$$

where $\boldsymbol{G}$ is the Gram matrix defined as $\boldsymbol{G}=\boldsymbol{A}^{H} \boldsymbol{A}$. Thus, we propose an algorithm to decrease the mutual coherence of $A$ which is determined by minimizing the whole elements of $\boldsymbol{G}$ except the main diagonal elements.

\subsection{Measurement matrix design for 1D-CS model}

As mentioned before, among the four cases we discussed, cases 1 and 2 cannot be converted to 2D model. We consider the optimization of these two cases as measurement matrix design for 1D-CS model.

Therefore, the following cost function can be defined for our problem:

$$
\begin{aligned}
C & =\left\|\boldsymbol{G}-\boldsymbol{I}_{N}\right\|_{F}^{2} \\
& =\operatorname{trace}\left\{\left(\boldsymbol{A}^{\boldsymbol{H}} \boldsymbol{A}-\boldsymbol{I}_{N}\right)\left(\boldsymbol{A}^{\boldsymbol{H}} \boldsymbol{A}-\boldsymbol{I}_{N}\right)^{H}\right\} .
\end{aligned}
$$

Thus, the optimization problem is formulated as $\hat{\boldsymbol{\Phi}}=\arg \min _{\boldsymbol{\Phi}} C$.

In our proposed method, first we optimize matrix $A$ using gradient descent algorithm [31], then the measurement matrix $\Phi$ is obtained form $\boldsymbol{A}$ by using least square (LS) estimator. In the gradient descent method, the gradient of cost function $C$ needs to be computed with respect to the unknown variable $A$, denoted as

$$
\nabla C=\frac{\partial C}{\partial A}=4 A\left(A^{H} \boldsymbol{A}-\boldsymbol{I}_{N}\right)
$$

then, the equation $\boldsymbol{A}=\boldsymbol{A}-\eta \nabla C$ is updated in an iterative process, where $\eta>0$ is the stepsize that can be fixed or updated iteratively by backtracking line search algorithm [35]. Before the execution of descent algorithm, the columns of matrix $\boldsymbol{A}$ need to be normalized as:

$$
\boldsymbol{a}_{i}=\boldsymbol{a}_{i} /\left\|\boldsymbol{a}_{i}\right\|_{2}
$$

The algorithm can be stopped when the stopping criterion $\|\nabla C\|_{F} \leq \varepsilon$ for a small and positive constant $\varepsilon$ or after a several number of iterations $\left(N_{G D}\right)$.

\subsubsection{Case 1}

After obtaining $\boldsymbol{A}$ for case 1, the measurement matrix of $p$ th pulse and $m$ th receiver $\left(\boldsymbol{\phi}_{p m}\right)$ is given by solving the following linear equation:

$$
\boldsymbol{A}_{p m}=\boldsymbol{\phi}_{p m} \boldsymbol{\psi}_{p m}
$$

where $A_{p m}$ is defined in (27). From Eqs. (6) to (13), it can be noted that some parts of matrix $\boldsymbol{\Psi}_{p m}$ is filled with 0 , and consequently $\boldsymbol{\psi}_{p m}^{H} \boldsymbol{\psi}_{p m}$ tends to be ill-conditioned. Therefore, the LS estimator which needs to calculate $\left(\boldsymbol{\psi}_{p m}^{H} \boldsymbol{\psi}_{p m}\right)^{-1}$ cannot be exploited, directly. To resolve this problem, we use the economy-size singular value decomposition (eSVD) of $\boldsymbol{\psi}_{p m} \in \mathbb{C}^{\tilde{L} \times N}$ given by

$$
\boldsymbol{\psi}_{p m}=\boldsymbol{U}_{1, p m}\left(\begin{array}{cc}
\boldsymbol{\Delta}_{1, p m} & 0 \\
0 & 0
\end{array}\right) \boldsymbol{D}_{1, p m}^{H}
$$

where $\boldsymbol{U}_{1, p m} \in \mathbb{C}^{\tilde{L} \times \tilde{L}}$ and $\boldsymbol{D}_{1, p m} \in \mathbb{C}^{N \times N}$ are unitary matrices, and $\Delta_{1, p m}=\operatorname{diag}\left(\lambda_{1, p m}, \ldots, \lambda_{r, p m}\right)$ is the singular values matrix of $\psi_{p m}$ that $\lambda_{i, p m} \neq 0$ for $i=1, \ldots, r$. In fact, $r$ is the number of non-zero singular values of $\boldsymbol{\psi}_{p m}$. The measurement matrix $\boldsymbol{\phi}_{p m}$ can be obtained as follows:

$$
\widehat{\boldsymbol{\Phi}}_{p m}=\left[\boldsymbol{U}_{1, p m}\left(\begin{array}{cc}
\boldsymbol{\Delta}_{1, p m}^{-1} & 0 \\
0 & 0
\end{array}\right) \boldsymbol{D}_{1, p m}^{H} \boldsymbol{A}_{p m}^{H}\right]^{H}
$$

Table 1 shows all the steps of the proposed method for optimizing measurement matrix in case 1 .

\subsubsection{Case 2}

Here, the measurement matrix is equal for all receivers. Therefore, we have redundant data that can be exploited to estimate $\boldsymbol{\phi}_{p}$.

In this case, we can reshape the equation $A_{p}=T_{p} \Psi_{p}$ to $\widetilde{\boldsymbol{A}}_{p}=\boldsymbol{\Phi}_{p} \widetilde{\Psi}_{p}$, where $\widetilde{\boldsymbol{A}}_{p} \in \mathbb{C}^{M \times N M_{r}}$ is the reshaped form of $\boldsymbol{A}_{p} \in \mathbb{C}^{M M_{r} \times N}$, and

$$
\widetilde{\boldsymbol{\Psi}}_{p}=\left[\boldsymbol{\psi}_{p 1} \boldsymbol{\psi}_{p 2} \ldots \boldsymbol{\psi}_{p M_{r}}\right] \in \mathbb{C}^{\widetilde{L}^{\times N M_{r}}}
$$

Now, the eSVD of $\widetilde{\Psi}_{p}$ is computed, and then $\boldsymbol{\phi}_{p}$ can be obtained similar to (39). 
Table 1 Designed measurement matrix algorithm for 1D model (case 1)

\footnotetext{
Input: Sparse representation basis $\Psi, \varepsilon$ for stopping criterion

Maximum number of iteration $N_{G D}$.

Output: Measurement matrix $\Phi$.

begin

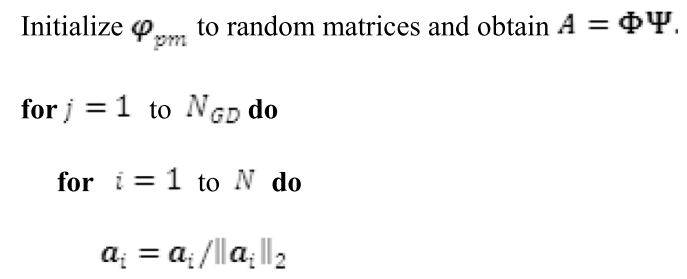

end

$\nabla C=4 A\left(A^{H} A-I_{N}\right)$

Calculate $\eta$ via backtracking line search.

$A \leftarrow A-\eta \nabla C$

Stopping criterion: quit if $\|\nabla C\|_{F} \leq \varepsilon$

end

Calculate eSVD for $\psi_{p m}$ :

$\psi_{p m}=U_{1, p m}\left(\begin{array}{cc}\Delta_{1 p m} & 0 \\ 0 & 0\end{array}\right) D_{1, p m}^{H}$

Estimate measurement matrix $\varphi_{m p}$ using LS estimator:

$$
\hat{\varphi}_{p m}=\left[U_{1, p m}\left(\begin{array}{cc}
\Delta_{1, p m}^{-1} & 0 \\
0 & 0
\end{array}\right) D_{1, p m}^{H} A_{p m}^{H}\right]^{H} .
$$

Normalized estimated measurement matrix $\hat{\varphi}_{\text {mm }}$ :$$
\hat{\varphi}_{p m} \leftarrow \hat{\varphi}_{p m} /\left\|\hat{\varphi}_{p m}\right\|_{F}
$$

Make the estimated measurement matrix $\tilde{\Phi}$ :

$$
\tilde{\Phi} \leftarrow \operatorname{Blkdiag}\left(\hat{\varphi}_{11}, \ldots, \hat{\varphi}_{p m}, \ldots, \hat{\varphi}_{N_{p} M}\right)
$$

End
}

\subsection{Measurement matrix design for 2D-CS model}

In our 2D-model, we have $\boldsymbol{A}=\boldsymbol{\Theta}^{T} \otimes \boldsymbol{B}$, where $\boldsymbol{B}=\boldsymbol{T} \boldsymbol{H}$. It is demonstrated in Appendix 2 that the MC of $A$ is equal to

$$
\mu(\mathbf{A})=\mu\left(\boldsymbol{\Theta}^{T}\right) \mu(\boldsymbol{B})
$$

According to (41), to optimize measurement matrix $\boldsymbol{T}$ based on minimizing $\mu(\mathbf{A})$ with respect to $T$, it is only needed to minimize $\mu(\boldsymbol{B})$ with respect to $\boldsymbol{T}$. Therefore, the optimization problem becomes:

$$
\hat{\boldsymbol{T}}=\arg \min _{\boldsymbol{T}}\left\|(\boldsymbol{T H})^{H} \boldsymbol{T} \boldsymbol{H}-\boldsymbol{I}_{N_{r} N_{a}}\right\|_{F}^{2}
$$

By using gradient descent optimization algorithm for cost function $C=\left\|\boldsymbol{B}^{H} \boldsymbol{B}-\boldsymbol{I}_{N_{r} N_{a}}\right\|_{F}^{2}$, we can obtain $\boldsymbol{B} \in \mathbb{C}^{M M_{r} \times N_{r} N_{a}}$ similar to $1 \mathrm{D}$ model.

\subsubsection{Case 3}

In this case, the measurement matrix is equal for all received pulses. After obtaining $\boldsymbol{B}$, the measurement matrix of $m$ th receiver $\left(\boldsymbol{\phi}_{m}\right)$ is calculated by solving the following linear equation:

$$
\boldsymbol{B}_{m}=\boldsymbol{\phi}_{m} \boldsymbol{H}_{m}
$$

where $\boldsymbol{B}=\left[\begin{array}{c}\boldsymbol{B}_{1} \\ \boldsymbol{B}_{2} \\ \vdots \\ \boldsymbol{B}_{M_{r}}\end{array}\right]$.

Similar to case 1, the eSVD of $\boldsymbol{H}_{m}$ is computed, and then $\phi_{m}$ is calculated.

\subsubsection{Case 4}

As noted for case 4, the measurement matrix is equal for all received pulses and receivers, i.e., $\boldsymbol{T}=\boldsymbol{I}_{M_{r}} \otimes \boldsymbol{\phi}$. Therefore, we can use the redundant data of all receivers in optimization of measurement matrix $\boldsymbol{\phi}$. To do so, the linear equation $\boldsymbol{B}=\boldsymbol{T} \boldsymbol{H}$ should be reshaped to $\widetilde{\boldsymbol{B}}=\boldsymbol{\phi} \tilde{\boldsymbol{H}}$, where $\widetilde{\boldsymbol{B}} \in \mathbb{C}^{M \times M_{r} N_{r} N_{a}}$ is the reshaped form of $\boldsymbol{B} \in \mathbb{C}^{M M_{r} \times N_{r} N_{a}}$, and

$$
\widetilde{\boldsymbol{H}}=\left[\boldsymbol{H}_{1} \boldsymbol{H}_{2} \ldots \boldsymbol{H}_{M_{r}}\right] \in \mathbb{C}^{\widetilde{L} \times M_{r} N_{r} N_{a}} .
$$

Now, the eSVD of $\widetilde{\boldsymbol{H}} \in \mathbb{C}^{\widetilde{L} \times M_{r} N_{r} N_{a}}$ is computed as follows:

$$
\widetilde{\boldsymbol{H}}=\boldsymbol{U}_{4}\left(\begin{array}{cc}
\boldsymbol{\Delta}_{4} & \mathbf{0} \\
\mathbf{0} & \mathbf{0}
\end{array}\right) \boldsymbol{D}_{4}^{H}
$$

where $\boldsymbol{U}_{4} \in \mathbb{C}^{\tilde{L} \times \tilde{L}}$ and $\boldsymbol{D}_{4} \in \mathbb{C}^{M_{r} N_{r} N_{a} \times M_{r} N_{r} N_{a}}$ are unitary matrices and $\Delta_{4}=\operatorname{diag}\left(\lambda^{\prime}{ }_{1}, \ldots, \lambda^{\prime}{ }_{r}\right)$ is the singular values matrix of $\mathbf{H}$ that $\lambda^{\prime}{ }_{i} \neq 0$ for $i=1, \ldots, r$. Also, $r$ is the number of non-zero singular values of $\boldsymbol{H}$. The measurement matrix $\phi$ can be given as follows:

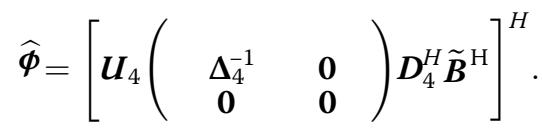

Table 2 shows all the steps of the proposed method to optimize measurement matrix in case 4 . In this table, $\boldsymbol{b}_{i}$ is the $i$ th column of $\boldsymbol{B}$. 
Table 2 Designed measurement matrix algorithm for 2D model case 4

Input: Sparse representation basis $H, \varepsilon$ for stopping criterion

Maximum number of iteration $N_{G D}$.

Output: Measurement matrix $T$.

begin

Initialize $\mathbf{T}$ to random matrix and obtain $B=T H$.

$$
\begin{gathered}
\text { for } j=1 \text { to } N_{G D} \text { do } \\
\text { for } i=1 \text { to }\left(N_{r} N_{O}\right) \text { do } \\
b_{i} \leftarrow b_{i} /\left\|b_{i}\right\|_{2}
\end{gathered}
$$

end

$\nabla C=4 B\left(B^{H} B-I_{N_{r} N_{0}}\right)$

Calculate $\eta$ via backtracking line search.

$B \leftarrow B-\eta \nabla C$

Stopping criterion: quit if $\|\nabla C\|_{F} \lesssim \varepsilon$

end

$$
\begin{aligned}
\operatorname{reshape}(\boldsymbol{B})=\hat{B} \in C^{M \times M_{\psi} N_{\psi} N_{0}} & \\
& \tilde{H}=\left[H_{1} H_{2} \ldots H_{M_{\psi}}\right]
\end{aligned}
$$

Calculate eSVD of $\tilde{H}$ :

$$
\tilde{H}=U_{4}\left(\begin{array}{cc}
\Delta_{4} & 0 \\
0 & 0
\end{array}\right) D_{4}^{H}
$$

Estimate measurement matrix $\varphi$ using LS estimator:

$\hat{\varphi}=\left[U_{4}\left(\begin{array}{cc}\Delta_{4}^{-1} & 0 \\ 0 & 0\end{array}\right) D_{4}^{H} \hat{B}^{H}\right]^{H}$

Normalized estimated measurement matrix $\hat{\varphi}$ :

$\hat{\varphi} \leftarrow \hat{\varphi} /\|\hat{\varphi}\|_{F}$

Make the estimated measurement matrix $\widehat{\mathrm{T}}$ :

$\widehat{\mathrm{T}} \leftarrow I_{M_{\psi}} \otimes \varphi$

End

\section{Computational complexity comparison}

The cases 1 and 2, called 1D proposed MMDGD methods, have almost the same computational complexity because the gradient descent algorithm for calculating matrix $A$ is the same for both cases. Also, the computational complexity of cases 3 and 4, called as 2D proposed MMDGD methods, are almost equal due to the similarity of their gradient descent algorithm. The main computational cost in each iteration of $1 \mathrm{D}$ and $2 \mathrm{D}$ proposed MMDGD methods belong to matrix product $\boldsymbol{A} \boldsymbol{A}^{H} \boldsymbol{A}$ and $\boldsymbol{B} \boldsymbol{B}^{H} \boldsymbol{B}$, respectively. The complexities of $\boldsymbol{A} \boldsymbol{A}^{H} \boldsymbol{A}$ and $\boldsymbol{B} \boldsymbol{B}^{H} \boldsymbol{B}$ are $O$ $\left(M^{2} M_{r}^{2} N_{p}^{2} N\right)$ and $O\left(M^{2} M_{r}^{2} N_{r} N_{a}\right)=O\left(M^{2} M_{r}^{2} N / N_{d}\right)$, respectively. Thus, the ratio of $2 \mathrm{D}-\mathrm{MMDGD}$ load over that of its $1 \mathrm{D}$ equivalent is $\frac{1}{N_{p}^{2} N_{d}}$.

The complexity of SCSM + SIR method that uses CVX package to optimize measurement matrix is $O\left(\widetilde{N}^{3}\right)$, where $\widetilde{N}=\widetilde{L}^{2}$ [35]. It can be approximated that $O\left(M^{2} M_{r}^{2} N_{r} N_{a}\right) \ll O\left(\widetilde{L}^{6}\right)$ due to the facts that $M \ll \widetilde{L}$, $N_{a} \approx N_{r}<\widetilde{L}$, and $M_{r}<\tilde{L}$ in our application. Therefore, the computational complexity of our proposed 2DMMDGD is much less than the SCSM + SIR method.

The computational complexity of $1 \mathrm{D}$ and $2 \mathrm{D}$ sparse recovery algorithms are discussed in [22] and [24]. As noted there, the computations of $1 \mathrm{D}$ and 2D algorithms for CS are $O\left(M M_{r} N_{p} N\right)$ and $O\left(M M_{r} N\right)+O\left(M M_{r} N_{p} N_{d}\right)$, respectively. Therefore, the ratio of $2 \mathrm{D}$ processing load over that of its $1 \mathrm{D}$ equivalent is $\frac{1}{N_{p}}+\frac{1}{N_{a} N_{r}}$.

In the next section, CPU time is used as a rough estimate of computational complexity of the algorithms.

\section{Simulation results}

For simulation, we consider a pulse-Doppler MIMO radar with $M_{t}$ transmit (TX) and $M_{r}$ receive $(\mathrm{RX})$ antennas having uniform linear array (ULA) with $\Delta_{t}=2.5 \lambda_{0}$ and $\Delta_{r}=0.5 \lambda_{0}$. The transmitted waveforms are obtained from efficient cyclic algorithm [36] that can produce sequences with very low auto- and cross-correlation sidelobes. We consider the length of sequence $L=32$ with unit power. The number of transmit and receive antennas are $M_{\mathrm{t}}=3$ and $M_{\mathrm{r}}=3$, respectively. The number of pulses is $N_{p}=3$. The carrier frequency, the pulse bandwidth, and PRF are $f_{c}=1 \mathrm{GHz}, B=10 \mathrm{MHz}$, and $f_{r}=2 \mathrm{kHz}$, respectively. The area under the radar includes $N_{r}=6$ range bins, $N_{d}=6$ Doppler bins, and $N_{a}=6$ angle bins between $0^{\circ}$ to $35^{\circ}$ with $7^{\circ}$ resolution.

We use the 1D-SLIM [15] and 2D-SLIM [22] algorithms to reconstruct $\hat{\boldsymbol{s}}$ and $\hat{\boldsymbol{S}}$ from the received compressed measurements in (17) and (23). Over 100 independent trials were run. In each trial, the locations of targets are generated randomly following a uniform random distribution.

The signal-to-noise ratio (SNR) is defined for each target located at the $(r, a, d)$ th range-angle-Doppler bin and the noise variance $\sigma^{2}$ as 


$$
\mathrm{SNR}=10 \log _{10}\left(\left|s_{r, a, d}\right|^{2} / \sigma^{2}\right) .
$$

The SNRs of all targets are considered equal.

At first, we compare the performance of four cases when the GRMM is used. In fact, GRMM is a complex Gaussian random matrix with zero mean and covariance matrix $\boldsymbol{I}$. The reconstruction error $\left(\|\hat{\boldsymbol{s}}-\boldsymbol{s}\|_{2}^{2}\right.$ $/\|\boldsymbol{s}\|_{2}^{2}$ ) for all cases versus the number of measurements, SNRs, and the number of pulses are shown in Fig. 2a-c, respectively, where $\hat{s}$ and $s$ are the estimated and true vectors containing the return complex reflection coefficients of targets, respectively. As seen in these figures, cases 1 and 3 have almost the same reconstruction error while outperforming two other cases.

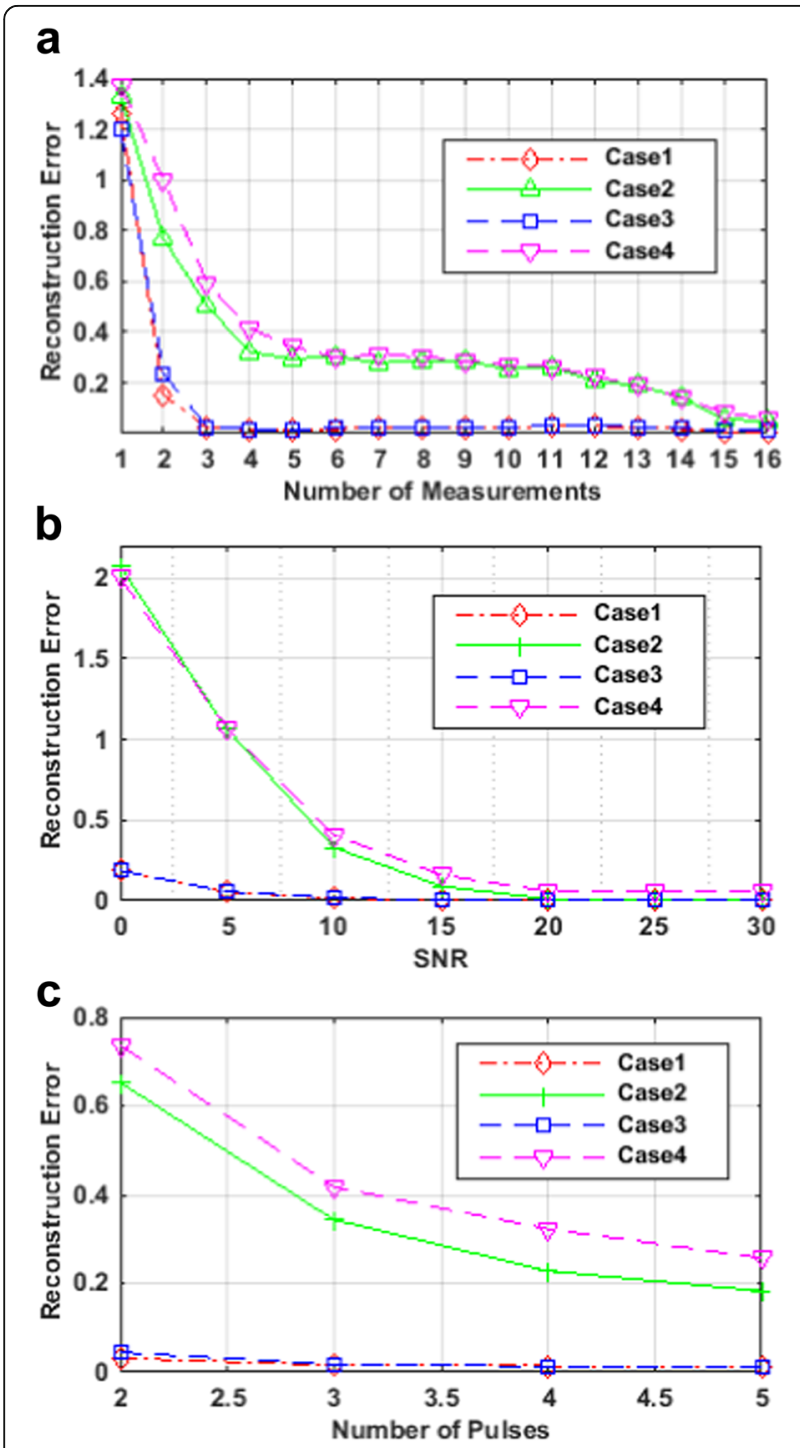

Fig. 2 Reconstruction error of four cases versus a the number of measurements, $\mathbf{b}$ SNRs, and $\mathbf{c}$ the number of pulses
It can be concluded from the simulation results that case 3 is the best choice for MIMO radar under the condition of low SNR, small number of measurements, and pulses. The reason is that the reconstruction error of case 3 is less than that of cases 2 and 4 while it is as small as the reconstruction error of case 1 . It should be noted that the computational cost of case 3 is much less than that of case 1 as shown in Section 5.

As depicted in these figures, by increasing the number of measurements, SNRs, and number of pulses, the reconstruction errors of cases 2 and 4 decrease and get close to cases 1 and 3 . As a result, under the condition of high SNR, large number of measurement, and pulses, case 4 can be considered for MIMO radar due to its ease of implementation. By the above discussion, one can easily find the effectiveness of our 2D proposed MMDGD methods compared to $1 \mathrm{D}$ ones.

The convergence of the gradient descent algorithm is demonstrated for convex and non-convex problems in [35] and [37, 38], respectively. Here, to show the convergence rate of the proposed method, we define the RMSE of convergence curve as $\|\boldsymbol{B}(j+1)-\boldsymbol{B}(j)\|_{F}^{2}$, where $j$ is the iteration index.

Figure 3 shows the convergence curve of this algorithm for our proposed measurement matrix design for 500 Monte Carlo runs. As it is clear, the proposed algorithm can converge after 20 iterations for $M=4$ and after 40 iterations for $M=8$.

Now, we compare the detection performance of the proposed method with GRMM, SIR, and SCSM + SIR methods using receiver operating characteristic (ROC) curve. Since in the SIR and SCSM + SIR methods, the measurement matrix is designed only for case 4 (because this matrix optimization will be very time consuming for

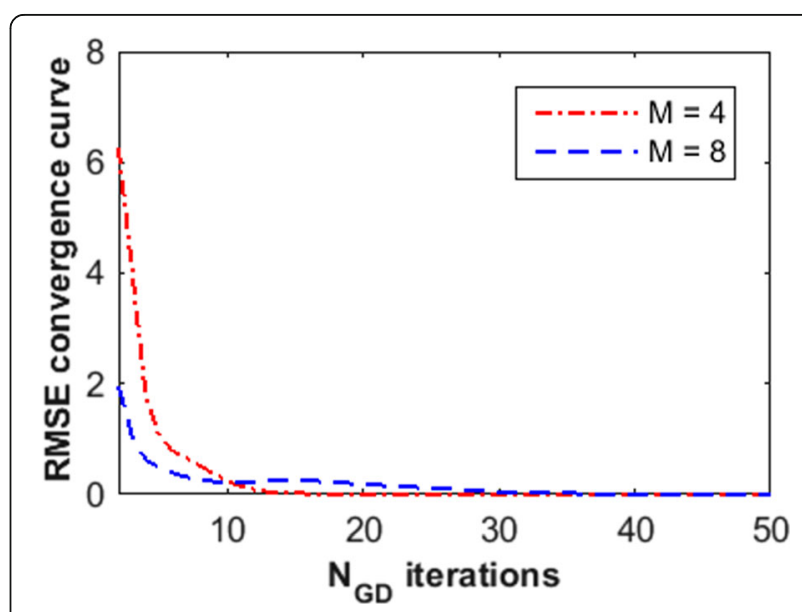

Fig. 3 Error of convergence versus the number of N_GD iterations 

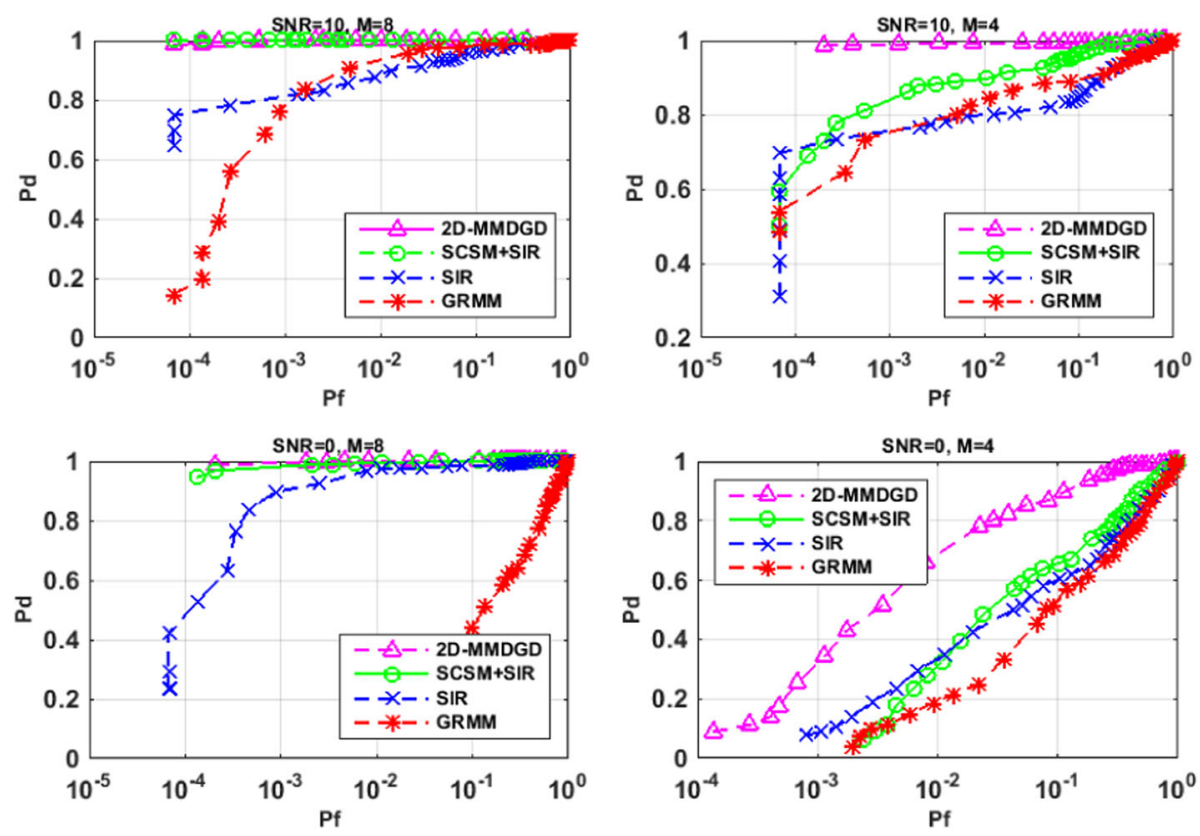

Fig. 4 Comparison of ROC curves for different measurement matrix in CS MIMO radar (resolution $7^{\circ}$ )

cases 1, 2, and 3 especially in SCSM + SIR), we consider only case 4 for comparison with these two methods.

In ROC curve, the probability of detection $\left(P_{\mathrm{d}}\right)$ is plotted versus the probability of false alarm $\left(P_{\mathrm{f}}\right)$. We consider any local maximum of the absolute value of $S$ that is larger than a selected threshold $\tau_{i}$ as a target. Then, we vary the threshold $\tau_{i}$ within an interval $\left[\begin{array}{ll}\tau_{L} & \tau_{H}\end{array}\right]$ and for each $\tau_{i}$, we count the number of detected actual or false targets. The empirical probability of detection $P_{\mathrm{d}}$ of actual targets and the empirical probability of false alarm $P_{\mathrm{f}}$ of false targets for different values of $\tau_{i}$ is obtained by repeating $N_{\text {itr }}$ trials of the experiment as:
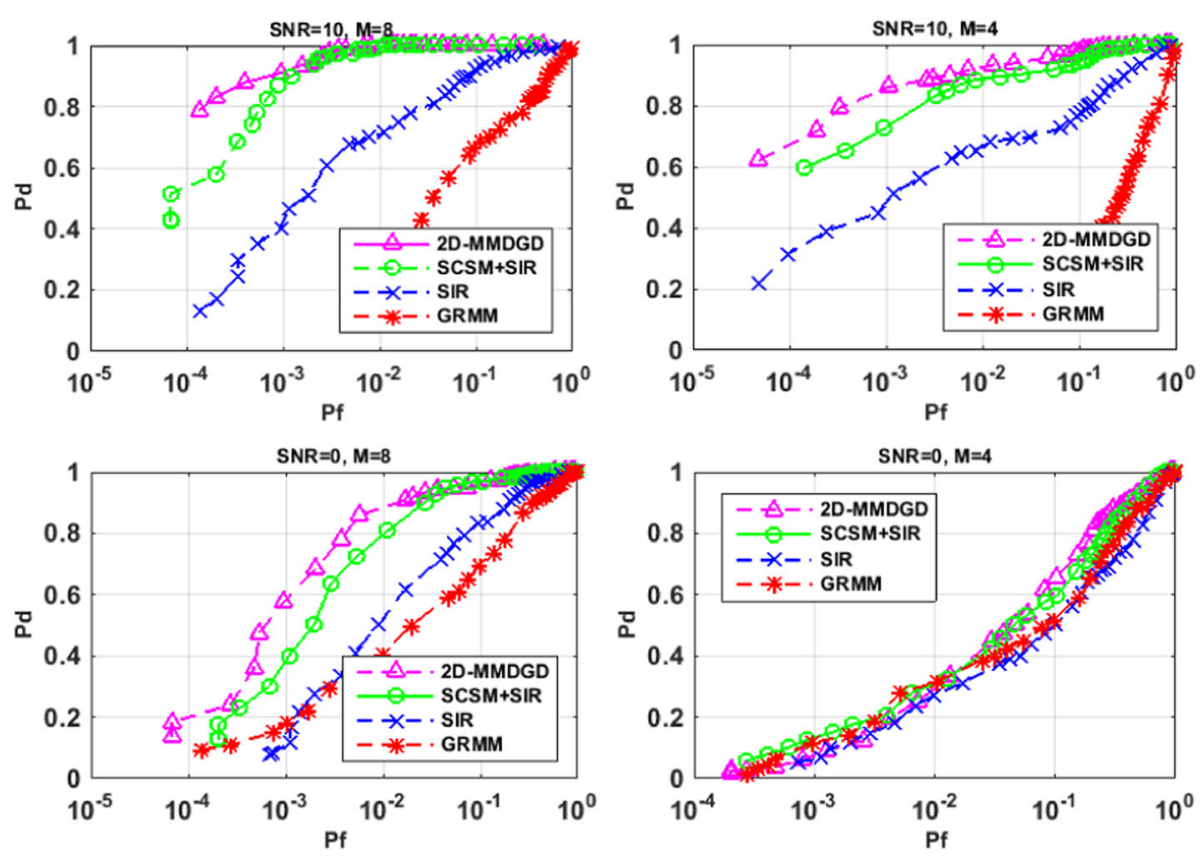

Fig. 5 Comparison of ROC curves of different measurement matrix in CS MIMO radar (resolution $2^{\circ}$ ) 


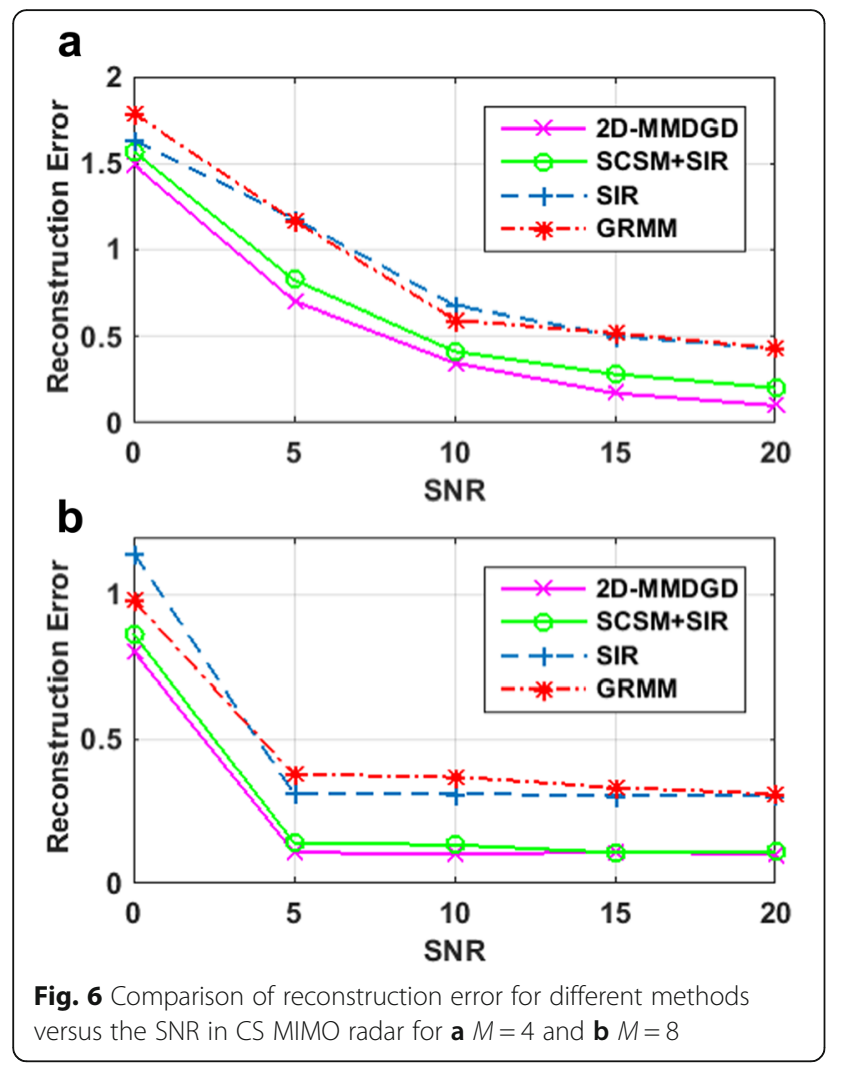

versus the SNR in CS MIMO radar for $\mathbf{a} M=4$ and $\mathbf{b} M=8$

$$
\begin{aligned}
& P_{\mathrm{d}}=\frac{\frac{1}{N_{\text {itr }}} \sum_{i=1}^{N_{\text {itr }}} n_{d i}}{N_{t}} \\
& P_{\mathrm{f}}=\frac{\frac{1}{N_{\text {itt }}} \sum_{i=1}^{N_{\text {itr }}} n_{f i}}{N-N_{t}}
\end{aligned}
$$

where $n_{d i}$ is the number of true targets and $n_{f i}$ is the number of false targets estimated at $i$ th iteration.

Figure 4 shows ROC curve at $\mathrm{SNR}=0$ and $10 \mathrm{~dB}, M=$ 8 and 4 , and $N_{t}=4$ with angle bins between $0^{\circ}$ to $35^{\circ}$ with $7^{\circ}$ resolution in CS MIMO radar. As it is obvious, 2D-MMDGD has the better performance compared to SIR, SIR + SCSM, and GRMM. The reason is that in the SIR method, only the signal-to-interference ratio is maximized and the increase of sparse recovery performance is not considered. On the other hand, in the SCSM + SIR method, to convert its non-convex problem to a convex one, some approximations are considered that lead to a sub-optimal solution, and thus, it may not have acceptable performance in all situations. Furthermore, the performance of our proposed method is always better than GRMM because the designed matrix has less mutual coherency compared to its initial value, i.e., GRMM.

Figure 5 shows ROC curve for angle bins between $0^{\circ}$ to $10^{\circ}$ with $2^{\circ}$ resolution and the same conditions as Fig. 4. As expected, by decreasing the resolution from 7 to $2^{\circ}$, the performance of all methods deteriorate due to the increase in MC. Also, our proposed method has better performance in low SNR and for lower number of measurements compared to other methods.

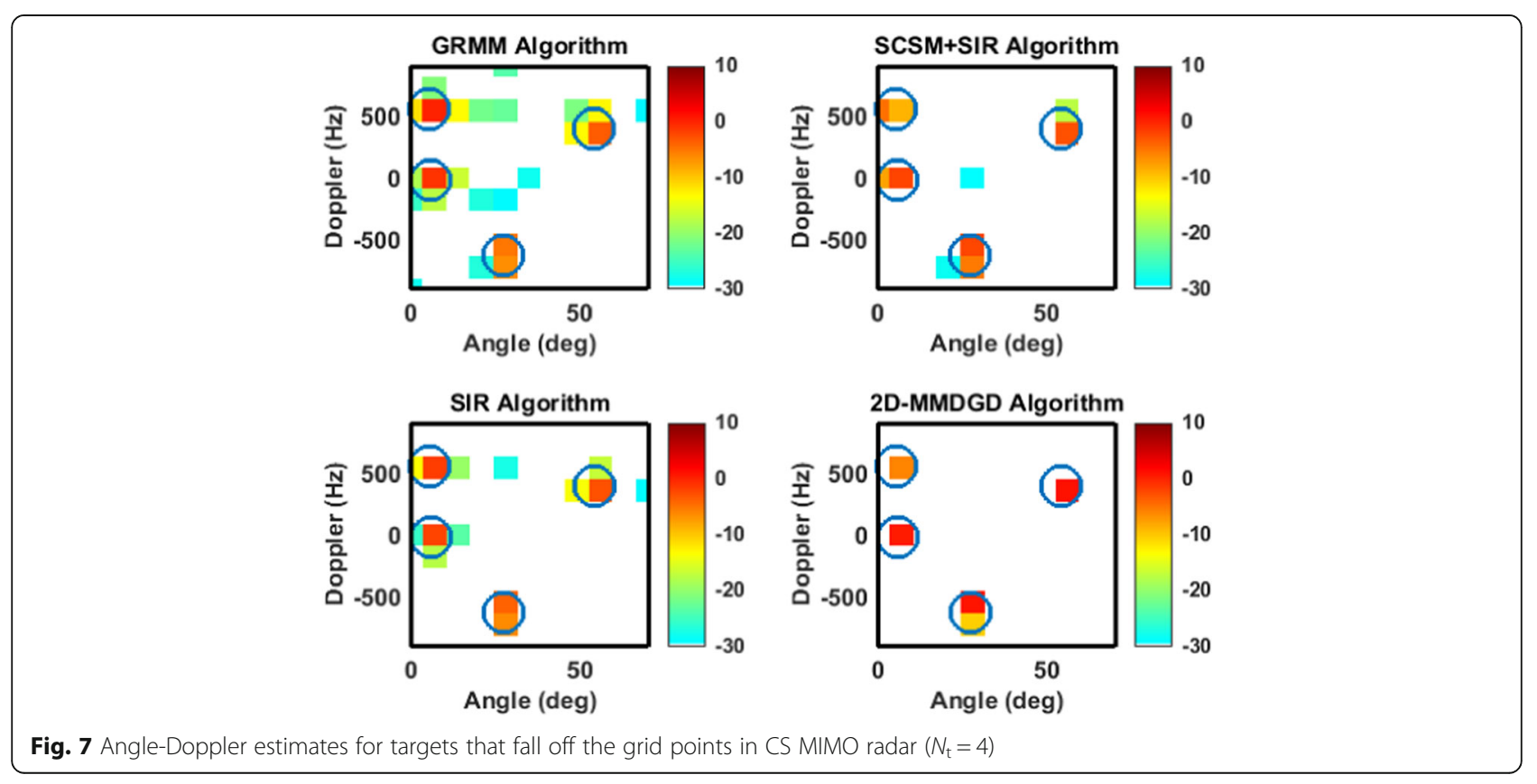



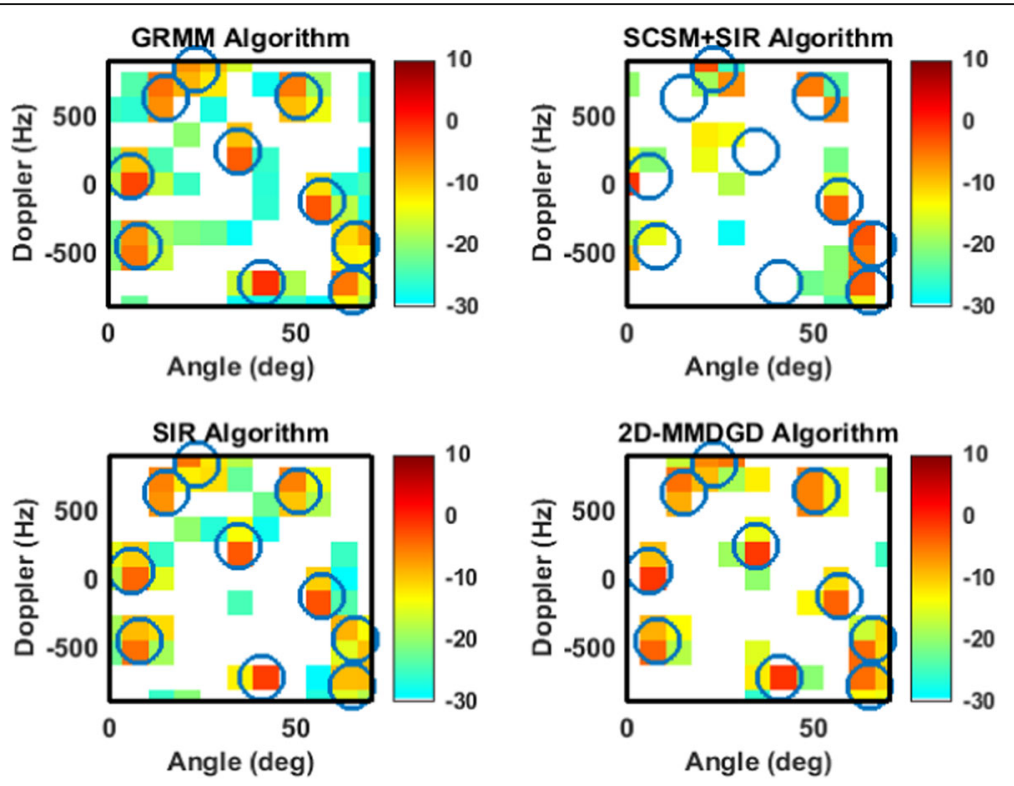

Fig. 8 Angle-Doppler estimates for targets that fall off the grid points in CS MIMO radar $\left(N_{t}=10\right)$

The reconstruction error versus the SNRs is shown in Fig. 6. As we see in Fig. 6a, the proposed method has better performance compared to other methods for small number of measurements in CS MIMO radar. Also, as depicted in Fig. 6b, our method and SCSM + SIR have nearly the same reconstruction error by increasing the number of measurements while outperforming SIR and GRMM methods.

The second scenario that we considered is the one that the targets fall off the grid points. In this scenario, a random non-integer multiple of the angle resolution and a random non-integer multiple of the Doppler resolution are chosen as the angle and Doppler of each target. For simulation, we consider $M=4, N_{p}=5, N_{d}=N_{a}=11, N_{r}$ $=6$ with a resolution of $7^{\circ}$.
The number of targets for Figs. 7 and 8 are $N_{t}=4$ and $N_{t}=10$, respectively. In these figures, the circles show the targets' true locations and the estimated amplitudes are shown with color-coded rectangles in decibel. It is demonstrated that our proposed method are able to capture the targets that fall off the grid points with lower sidelobes compared to other methods.

Figure 9 shows the runtime of measurement matrix design for different methods versus the number of measurements. The experiment is performed in MATLAB 8.1 environment using an Intel Core i7, $2.7 \mathrm{GHz}$ processor with $4 \mathrm{~GB}$ of memory, and under Microsoft windows 7 operating system. This figure demonstrates the effectiveness of our proposed 2D method, and it shows clearly that the 2D-MMDGD has much lower computational

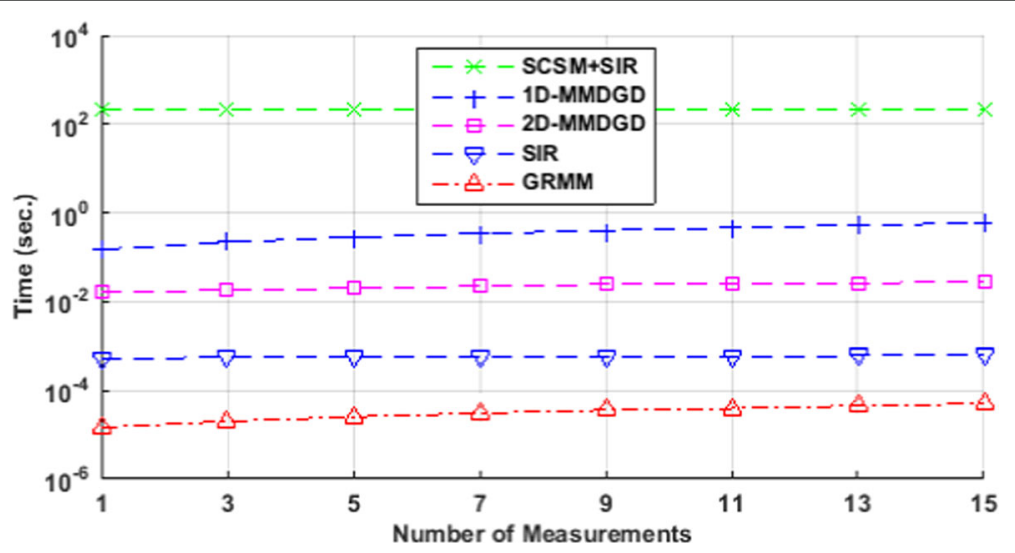

Fig. 9 Comparison of runtime of measurement matrix design for different methods versus the number of measurements in CS MIMO radar 
cost compared to the 1D one. Also, as explained in Section 5, SCSM + SIR has more runtime compared to our proposed 2D-MMDGD, and also the other methods.

\section{Conclusions}

We have introduced a new 1D and 2D CS model for a pulse-Doppler collocated MIMO radar. Then, we divided the measurement matrix design into four cases in which the measurement matrices applied to receivers and pulses can be equal or different. The measurement matrix design for all cases was proposed based on minimizing MC of sensing matrix using gradient descent algorithm. Looking at the performance comparison between $1 \mathrm{D}$ and $2 \mathrm{D}$ methods shows that case 3 can be the algorithm of choice for practical CS MIMO radar systems. The simulation results show that our proposed 2D-MMDGD, even in case 4 scenario outperform GRMM, SIR, and SCSM + SIR methods while having much lower computations.

\section{Appendix 1}

8.1 The statistical analysis of MC of sensing matrix A:

In this appendix, we analyze the $\mathrm{MC}$ of four cases from a statistical point of view. To do so, we obtain the mean and variance of $\mathrm{MC}$ for all cases and then compare their results. Let the components of measurement matrix $\boldsymbol{\phi}_{p m}$ be considered as random variable.

The MC of $\boldsymbol{A}$ is proportional to $\mu(\boldsymbol{A}) \propto \max _{i \neq j} \rho_{i, j}$, where $\rho_{i, j}$ can be given as:

$$
\rho_{i, j}=\left|<\boldsymbol{a}_{i}, \boldsymbol{a}_{j}>\right| \text { for } i, j=1,2, \ldots, N
$$

where

$$
\begin{aligned}
& \boldsymbol{a}_{i}=\sum_{p}^{N_{p}} \sum_{m}^{M_{r}} \boldsymbol{\phi}_{p m} \boldsymbol{\Psi}_{p m}(:, i) \\
& \boldsymbol{\psi}_{p m}(:, i)=e^{j 2 \pi\left(-\frac{1}{2}+\left(d_{i}-1\right) / N_{d}\right)(p-1)} e^{-j \pi \sin \left(a_{i}\right)(m-1)} \boldsymbol{J}_{r_{i}} \boldsymbol{V} \boldsymbol{a}_{a_{i}}
\end{aligned}
$$

Now, $\rho_{i, j}$ can be extended as:

$$
\begin{aligned}
\rho_{i, j} & =\left|\sum_{p}^{N_{p}} \sum_{m}^{M_{r}} e^{j\left(\frac{2 \pi\left(d_{j}-d_{i}\right)}{N_{d}}\right)(p-1)} e^{j \pi\left(\sin \left(a_{i}\right)-\sin \left(a_{i j}\right)\right)(m-1)} \boldsymbol{a}_{a_{i}}^{H} \boldsymbol{V} \boldsymbol{J}_{r_{i}}^{H} \boldsymbol{\phi}_{p m}^{H} \boldsymbol{\phi}_{p m} \boldsymbol{J} \boldsymbol{J}_{r_{j}} \boldsymbol{V} \boldsymbol{a}_{a_{j}}\right| \\
& =\left|\sum_{p}^{N_{p}} \sum_{m}^{M_{r}} C_{p, i j} C_{m, i j} \delta_{p m, i j}\right|
\end{aligned}
$$

where

$$
\begin{aligned}
& \delta_{p m, i j}=\boldsymbol{a}_{a_{i}}^{H} \boldsymbol{V} \boldsymbol{J}_{r_{i}}^{H} \boldsymbol{\phi}_{p m}^{H} \boldsymbol{\phi}_{p m} \boldsymbol{J}_{r_{j}} \boldsymbol{V} \boldsymbol{a}_{a_{j}} \\
& C_{p, i j}=e^{j\left(\frac{2 \pi\left(d_{j}-d_{i}\right)}{N_{d}}\right)(p-1)}
\end{aligned}
$$

$$
C_{m, i j}=e^{j \pi\left(\sin \left(a_{i}\right)-\sin \left(a_{j}\right)\right)(m-1)} .
$$

Since the statistical properties of measurement matrix $\boldsymbol{\phi}_{p m}$ is independent of the number of pulses and antennas, and also it can be approximately independent of $(i, j)$ as shown in [32], we can conclude that the mean and variance of $\delta_{p m, i j}$ for all cases are equal, i.e.,

$$
\begin{aligned}
& E\left(\delta_{p m}\right)=E\left(\delta_{p}\right)=E\left(\delta_{m}\right)=E(\delta) . \\
& \operatorname{var}\left(\delta_{p m}\right)=\operatorname{var}\left(\delta_{p}\right)=\operatorname{var}\left(\delta_{m}\right)=\operatorname{var}(\delta)=\vartheta^{2} .
\end{aligned}
$$

Therefore, we have

$$
E\left(\mu_{\text {case 1 }}\right)=E\left(\mu_{\text {case 2 }}\right)=E\left(\mu_{\text {case 3 }}\right)=E\left(\mu_{\text {case } 4}\right) .
$$

Now, we analyze the variance of MC for all cases. Variance analysis of MC for case 1:

$$
\begin{aligned}
\sigma_{\rho_{i, j}^{\text {case } 1}}^{2} & =\operatorname{var}\left(\sum_{p}^{N_{p}} \sum_{m}^{M_{r}} C_{p, i j} C_{m, i j} \delta_{p m}\right) \\
& =\sum_{p}^{N_{p}} \sum_{m}^{M_{r}}\left|C_{p, i j}\right|^{2}\left|C_{m, i j}\right|^{2} \operatorname{var}\left(\delta_{p m}\right) .
\end{aligned}
$$

Due to $\left|C_{p, i j}\right|=1$ and $\left|C_{m, i j}\right|=1$, we have

$$
\operatorname{var}\left(\mu_{\text {case 1 }}\right)=N_{p} M_{r} \vartheta^{2} \text {. }
$$

Variance analysis of MC for case 2:

$$
\begin{aligned}
\sigma_{\rho_{i, j}^{\text {case }}}^{2} & =\operatorname{var}\left(\sum_{p}^{N_{p}} \sum_{m}^{M_{r}} C_{p, i j} C_{m, i j} \delta_{p}\right) \\
& =\left|\sum_{m}^{M_{r}} C_{m}\right| \sum_{p}^{2}\left|C_{p}\right|^{2} \operatorname{var}\left(\delta_{p}\right) \\
& =\left|\sum_{m}^{N_{r}} e^{j \pi\left(\sin \left(a_{i}\right)-\sin \left(a_{j}\right)\right)(m-1)}\right|^{2} N_{p} \vartheta^{2} \\
& =\left|\frac{\sin \left(\frac{1}{2} M_{r} \pi\left(\sin \left(a_{i}\right)-\sin \left(a_{j}\right)\right)\right)}{\sin \left(\frac{1}{2} \pi\left(\sin \left(a_{i}\right)-\sin \left(a_{j}\right)\right)\right)}\right|^{2} N_{p} \vartheta^{2} .
\end{aligned}
$$

Due to $\left|C_{p, i j}\right|=1$, and $\max \left(\left|\sum_{m}^{M_{r}} C_{m}\right|^{2}\right)=M_{r}^{2}$, we have

$$
\operatorname{var}\left(\mu_{\text {case 2 }}\right)=\max _{i, j}\left(\operatorname{var}\left(\rho_{i, j}\right)\right)=N_{p} M_{r}^{2} \vartheta^{2} .
$$

Variance analysis of MC for case 3: 


$$
\begin{aligned}
\sigma_{\rho_{i, j}^{\text {case } 3}}^{2} & =\operatorname{var}\left(\sum_{p}^{N_{p}} \sum_{m}^{M_{r}} C_{p, i j} C_{m, i j} \delta_{m}\right) \\
& =\left|\sum_{p}^{N_{p}} C_{p}\right| \sum_{m}^{M_{r}}\left|C_{m}\right|^{2} \operatorname{var}\left(\delta_{m}\right) \\
& =\left|\sum_{p}^{N_{p}} e^{j\left(\frac{2 \pi\left(d_{j}-d_{i}\right)}{N_{d}}\right)(p-1)}\right|^{2} M_{r} \vartheta^{2} \\
& =\left|\frac{\sin \left(N_{p} \frac{\pi\left(d_{j}-d_{i}\right)}{N_{d}}\right)}{\sin \left(\frac{\pi\left(d_{j}-d_{i}\right)}{N_{d}}\right)}\right|^{2} M_{r} \vartheta^{2}
\end{aligned}
$$

$$
\begin{aligned}
\mu(\boldsymbol{A})= & \max _{i \neq j} \frac{\rho_{i, j}}{\left\|\boldsymbol{a}_{i}\right\|_{2}\left\|\boldsymbol{a}_{j}\right\|_{2}}=\max _{i \neq j} \frac{\left|\boldsymbol{a}_{i}^{H} \boldsymbol{a}_{j}\right|}{\left\|\boldsymbol{a}_{i}\right\|_{2}\left\|\boldsymbol{a}_{j}\right\|_{2}} \\
= & \max _{\substack{d \neq d^{\prime} \\
n \neq n^{\prime}}} \frac{\left|\left(\left((\boldsymbol{\Theta})_{d}^{r}\right)^{T} \otimes(\boldsymbol{B})_{n}^{c}\right)^{H}\left(\left((\boldsymbol{\Theta})_{d^{\prime}}^{r}\right)^{T} \otimes(\boldsymbol{B})_{n^{\prime}}^{c}\right)\right|}{\left.\|(\boldsymbol{\Theta})_{d}^{r}\right)^{T} \otimes(\boldsymbol{B})_{n}^{c}\left\|_{2}\right\|\left((\boldsymbol{\Theta})_{d^{\prime}}^{r}\right)^{T} \otimes(\boldsymbol{B})_{n^{\prime}}^{c} \|_{2}} \\
= & \max _{d \neq d^{\prime}} \frac{\left|\left((\boldsymbol{\Theta})_{d}^{r}\right)^{H} \otimes(\boldsymbol{\Theta})_{d^{\prime}}^{r}\right|}{\left\|(\boldsymbol{\Theta})_{d}^{r}\right\|_{2}\left\|(\boldsymbol{\Theta})_{d}^{r}\right\|_{2}} \max _{n \neq n^{\prime}} \frac{\left|\left((\boldsymbol{B})_{n}^{c} H \otimes(\boldsymbol{B})_{n^{\prime}}^{c}\right)\right|}{\left\|(\boldsymbol{B})_{n}^{c}\right\|_{2}\left\|(\boldsymbol{B})_{n^{\prime}}^{c}\right\|_{2}} \\
= & \mu\left(\boldsymbol{\Theta}^{T}\right) \mu(\boldsymbol{B})
\end{aligned}
$$

Due to $\left|C_{m, i j}\right|=1$ and $\max \left(\left|\sum_{p}^{N_{p}} C_{p}\right|^{2}\right)=N_{p}^{2}$, we have

$$
\operatorname{var}\left(\mu_{\text {case 3 }}\right) \propto \max _{i, j}\left(\operatorname{var}\left(\rho_{i, j}\right)\right)=N_{p}^{2} M_{r} \vartheta^{2} \text {. }
$$

Variance analysis of MC for case 4:

$$
\begin{aligned}
\sigma_{\rho_{i, j}^{\text {case } 4}}^{2} & =\operatorname{var}\left(\sum_{p}^{N_{p}} \sum_{m}^{M_{r}} C_{p} C_{m} \delta\right)=\left|\sum_{p}^{N_{p}} C_{p}\right|^{2}\left|\sum_{m}^{M_{r}} C_{m}\right|^{2} \operatorname{var}(\delta) \\
& =\left|\frac{\sin \left(N_{p} \frac{\pi\left(d_{j}-d_{i}\right)}{N_{d}}\right)}{\sin \left(\frac{\pi\left(d_{j}-d_{i}\right)}{N_{d}}\right)}\right|^{2}\left|\frac{\sin \left(\frac{1}{2} M_{r} \pi\left(\sin \left(a_{i}\right)-\sin \left(a_{j}\right)\right)\right)}{\sin \left(\frac{1}{2} \pi\left(\sin \left(a_{i}\right)-\sin \left(a_{j}\right)\right)\right)}\right|^{2} \vartheta^{2} .
\end{aligned}
$$

Due to $\max \left(\left|\sum_{p}^{N_{p}} C_{p}\right|^{2}\right)=N_{p}^{2}$ and $\max \left(\left|\sum_{m}^{M_{r}} C_{m}\right|^{2}\right)=M_{r}^{2}$, we have

$$
\operatorname{var}\left(\mu_{\text {case } 4}\right) \propto \max _{i, j}\left(\operatorname{var}\left(\rho_{i, j}\right)\right)=N_{p}^{2} M_{r}^{2} \vartheta^{2} .
$$

\section{Appendix 2}

9.1 The relationship between the MC of $A, B$, and $\Theta$ :

Consider the following Kroneker product properties [33]:

- if $\boldsymbol{A}=\boldsymbol{\Theta}^{T} \otimes \boldsymbol{B}$, then $\boldsymbol{a}_{i}=\left((\boldsymbol{\Theta})_{d}^{r}\right)^{T} \otimes(\boldsymbol{B})_{n}^{c}$, where $\boldsymbol{a}_{i}$ is the $i$ th column of $\mathbf{A},(\boldsymbol{\Theta})_{d}^{r}$ is $d$ th row of $\boldsymbol{\Theta}$, and $(\boldsymbol{B})_{n}^{c}$ is the $n$th column of matrix $\boldsymbol{B}$.

- $\left\|\left((\boldsymbol{\Theta})_{d}^{r}\right)^{T} \otimes(\boldsymbol{B})_{n}^{c}\right\|_{2}=\left\|(\boldsymbol{\Theta})_{d}^{r}\right\|_{2}\left\|(\boldsymbol{B})_{n}^{c}\right\|_{2}$

- $\left|\left(\left((\boldsymbol{\Theta})_{d}^{r}\right)^{T} \otimes(\boldsymbol{B})_{n}^{c}\right) H\left(\left((\boldsymbol{\Theta})_{d^{\prime}}^{r}\right)^{T} \otimes(\boldsymbol{B})_{n^{\prime}}^{c}\right)\right|$

$$
=\left|\left((\boldsymbol{\Theta})_{d}^{r}\right)^{H} \otimes(\boldsymbol{\Theta})_{d^{\prime}}^{r}\right|\left|\left((\boldsymbol{B})_{n}^{c}\right)^{H} \otimes(\boldsymbol{B})_{n^{\prime}}^{c}\right|
$$

Then, the $\mathrm{MC}$ of $\mathbf{A}$ is obtained as, where $d, d^{\prime}=1, \ldots, N_{d}$ and $n, n^{\prime}=1, \ldots, N_{r} N_{a}$. Also, we have $i=(d-1) N_{r} N_{a}+n$ and $j=\left(d^{\prime}-1\right) N_{r} N_{a}+n^{\prime}$.

\section{Acknowledgements}

The authors would like to thank the Referees for the careful revision of the paper.

Authors' contributions

All the authors have contributed to the study conception and design of algorithms, theoretical analysis and simulations, drafting of manuscript, and critical revision. All authors read and approved the final manuscript.

\section{Authors' information}

Nafiseh Shahbazi received a B.S. degree in 2008 and an M.S. degree in 2008 in Electrical Engineering at Amirkabir University of Technology, Tehran, Iran. She is currently working for a Ph.D. degree in the School of Electrical and Computer Engineering, University of Tehran, from 2011. Her current research interests are in the areas of array signal processing, radar signal processing, and compressive sensing.

Aliazam Abbasfar received a B.Sc. (Highest Honors) and a M.Sc. degree in Electrical Engineering at the University of Tehran, Iran, in 1992 and 1995 and a Ph.D. degree in Electrical Engineering at the University of California Los Angeles (UCLA) in 2005. Between 2001 and 2004, he held a position as a senior design engineer in the areas of communication system design and digital VLSI/ASIC design with various start-up companies in California. Upon graduation from the UCLA, he joined Rambus Inc. where he was a Principal Engineer working on high-speed data communications on wireline serial and parallel links. He is currently an Assistant Professor at the University of Tehran. Mohammad Jabbarian-Jahromi received a Ph.D degree in Electrical Engineering at Iran University of Science and Technology, Tehran, Iran, in 2016. His current research interests are in the areas of array signal processing, radar signal processing, and sparse reconstruction.

\section{Competing interests}

The authors declare that they have no competing interests.

\section{Author details}

${ }^{1}$ School of Electrical and Computer Engineering, University of Tehran, Tehran, Iran. ${ }^{2}$ School of Electrical Engineering, Iran University of Science and Technology, Tehran, Iran.

Received: 27 August 2016 Accepted: 21 January 2017

Published online: 01 March 2017

\section{References}

1. DL Donoho, Compressed sensing. IEEE Trans Inf Theory 52(4), 1289-1306 (2006)

2. EJ Candes, MB Wakin, An introduction to compressive sampling (a sensing/ sampling paradigm that goes against the common knowledge in data acquisition). IEEE Signal Process Mag. 25(2), 21-30 (2008) 
3. M Mishali, Y Eldar, Blind multi-band signal reconstruction: compressed sensing for analog signals. IEEE Trans Signal Process. 57(30), 993-1009 (2009)

4. J Ma, FX Le Dimet, Deblurring from highly incomplete measurements for remote sensing. IEEE Trans Geosci Remote Sens. 47(3), 792-802 (2009)

5. SF Cotter, BD Rao, Sparse channel estimation via matching pursuit with application to equalization. IEEE Trans Commun. 50(3), 374-377 (2002)

6. J Trzasko, A Manduca, E Borisch, Highly under sampled magnetic resonance image reconstruction via homotopic ell-0-minimization. IEEE Trans Med Imaging 28(1), 106-121 (2009)

7. J Romberg, Imaging via compressive sampling (introduction to compressive sampling and recovery via convex programming). IEEE Signal Process Mag. 25(2), 14-20 (2008)

8. S Chen, D Donoho, M Saunders, Atomic decomposition by basis pursuit. SIAM J Sci Comput. 20, 33-61 (1998)

9. R Baraniuk, P Steeghs, Compressive radar imaging, in Process. Radar Conf, 2007, pp. 128-133

10. AC Gurbuz, JH McClellan, WR Scott, Compressive sensing for GPR imaging in Proc. IEEE, 41st Asilomar Conf. Signals, Syst. Computer, Pacific Grove, CA, 2007, pp. 2223-2227.

11. M Herman, T Strohmer, Compressed sensing radar. in Proc. IEEE Int. Conf. IEEE, Acoustic Speech Signal Process., Las Vegas, NV, 2008, pp. 2617-2620

12. AC Gurbuz, JH McClellan, V Cevher, A compressive beamforming method. in Proc. IEEE Int. Conf. Acoustic Speech Signal Process, Las Vegas, NV, 2008, pp. $2617-2620$

13. AM Haimovich, RS Blum, LJ Cimini, MIMO radar with widely separated antennas. IEEE Signal Process Mag. 25(1), 116-129 (2008)

14. P Stoica, J Li, MIMO radar with collocated antennas. IEEE Signal Process Mag. 24(5), 106-114 (2007)

15. X Tan, W Roberts, J Li, P Stoica, Sparse learning via iterative minimization with application to MIMO radar imaging. IEEE Trans Signal Process. 59(3), 1088-1101 (2011)

16. T Yardibi, J Li, P Stoica, M Xue, AB Baggeroer, Source localization and sensing: a nonparametric iterative adaptive approach based on weighted least squares. IEEE Trans Aerosp Electron Syst. 46, 425-443 (2010)

17. M Jabbarian-Jahromi, MH Kahaei, Two-dimensional sparse solution for bistatic MIMO radars in presence of jammers. 22nd Iranian Conference on Electrical Engineering (ICEE), pp. 1755-1759. IEEE, Tehran (2014)

18. Q Hou, Y Liu, Z Chen, Sh Su, Sparse radar imaging using 2D compressed sensing. Proc. SPIE 9252, Millimetre Wave and Terahertz Sensors and Technology VII, Oct. 2014, 9252

19. M Jabbarian-Jahromi, MH Kahaei, Two-dimensional iterative adaptive approach for a sparse matrix solution. IET Electron Lett 50(1), 45-47 (2014)

20. A Ghaffari, M Babaie-Zadeh, C Jutten, Sparse decomposition of two dimensional signals. in IEEE International Conf. on Acoustics, Speech and Signal Process., ICASSP 2009, pp. 3157-3160. IEEE, Taipei (2009)

21. HM Hyder, K Mahata, Range-Doppler imaging via sparse representation, in IEEE Radar Conf. (RADAR), 2011, pp. 486-491

22. M Jabbarian-Jahromi, MH Kahaei, Two-dimensional SLIM with application to pulse Doppler MIMO radars. EURASIP J Adv Signal Process. 69, 45-47 (2015)

23. M Jabbarian-Jahromi, MH Kahaei, Complex two-dimensional TNIPM for $\mathrm{I}_{1}$ norm-based sparse optimization to collocated MIMO radar. IEEJ Trans Electr Electron Eng. 11(2), 228-235 (2016)

24. M Jabbarian-Jahromi, N Shahbazi, MH Kahaei, A Abbasfar, Fast two-dimensional sparse Bayesian learning with application to pulse Doppler multiple-inputmultiple-output radars, in IET Radar, Sonar \& Navigation, 2015, pp. 1-10

25. G Chen, D Li, J Zhang, Iterative gradient projection algorithm for twodimensional compressive sensing sparse image reconstruction. Elsevier Signal Process. 104, 15-26 (2014)

26. Y Yu, AP Petropulu, HV Poor, MIMO radar using compressive sampling. IEEE J Selected Signal Process. 4(1), 146-163 (2010)

27. T Strohmer, B Friedlander, Compressed sensing for MIMO radar_algorithms and performance. in Proc. IEEE, $43^{\text {rd }}$ Asilomar Conf. Signals, Syst. Computer, Pacific Grove, CA, 2009, pp. 464-468

28. DL Donoho, X Huo, Uncertainty principles and ideal atomic decomposition. IEEE Trans Inform Theory 47(7), 2845-2862 (2001)

29. M Elad, Optimized projections for compressed sensing. IEEE Trans Signal Process. 55(12), 5695-5702 (2007)

30. JM Duarte-Carvajalino, G Sapiro, Learning to sense sparse signals: simultaneous sensing matrix and sparsifying dictionary optimization. IEEE Trans Image Process. 18(7), 1395-1408 (2009)
31. V Abolghasemi, S Ferdowsi, B Makkiabadi, S Sanei, On optimization of the measurement matrix for compressive sensing, in Process. European Signal Process. Conf, 2010, pp. 427-431

32. Y Yu, AP Petropulu, HV Poor, Measurement matrix design for compressive sensing-based MIMO radar. IEEE Trans Signal Process. 59(11), 5338-5352 (2011)

33. A George, F Seber, A Matrix Handbook for Statisticians (Wiley, Hoboken, 2008)

34. JA Tropp, Greed is good: algorithmic results for sparse approximation. IEEE Trans Inf Theory 50(10), 2231-2242 (2004)

35. S Boyd, L Vandenberghe, Convex Optimization (Cambridge University Press, Cambridge, 2004)

36. P Stocia, $\mathrm{H} \mathrm{He}, \mathrm{J} \mathrm{Li}$, New algorithms for designing unimodular sequences with good correlation properties. IEEE Trans Signal Process. 57(4), 14151425 (2009)

37. C Cartis, NIM Gould, PL Toint, On the complexity of steepest descent, Newton's and regularized Newton's methods for nonconvex unconstrained optimization. SIAM J Optim. 20(6), 2833-2852 (2010)

38. C. D. Sa, K. Olukotun, and C., Global convergence of stochastic gradient descent for some non-convex matrix problems. Proceedings of the 32nd International Conference on Machine Learning (ICML-15), 2015.

\section{Submit your manuscript to a SpringerOpen ${ }^{\circ}$ journal and benefit from:}

- Convenient online submission

- Rigorous peer review

- Immediate publication on acceptance

- Open access: articles freely available online

- High visibility within the field

- Retaining the copyright to your article

Submit your next manuscript at $>$ springeropen.com 